\title{
Ocean Shipping Container Availability Report
}

United States

Department

of Agriculture

A weekly publication of the Transportation Services Division, AMS

Agricultural

Marketing Service

\section{United States Department of Agriculture}
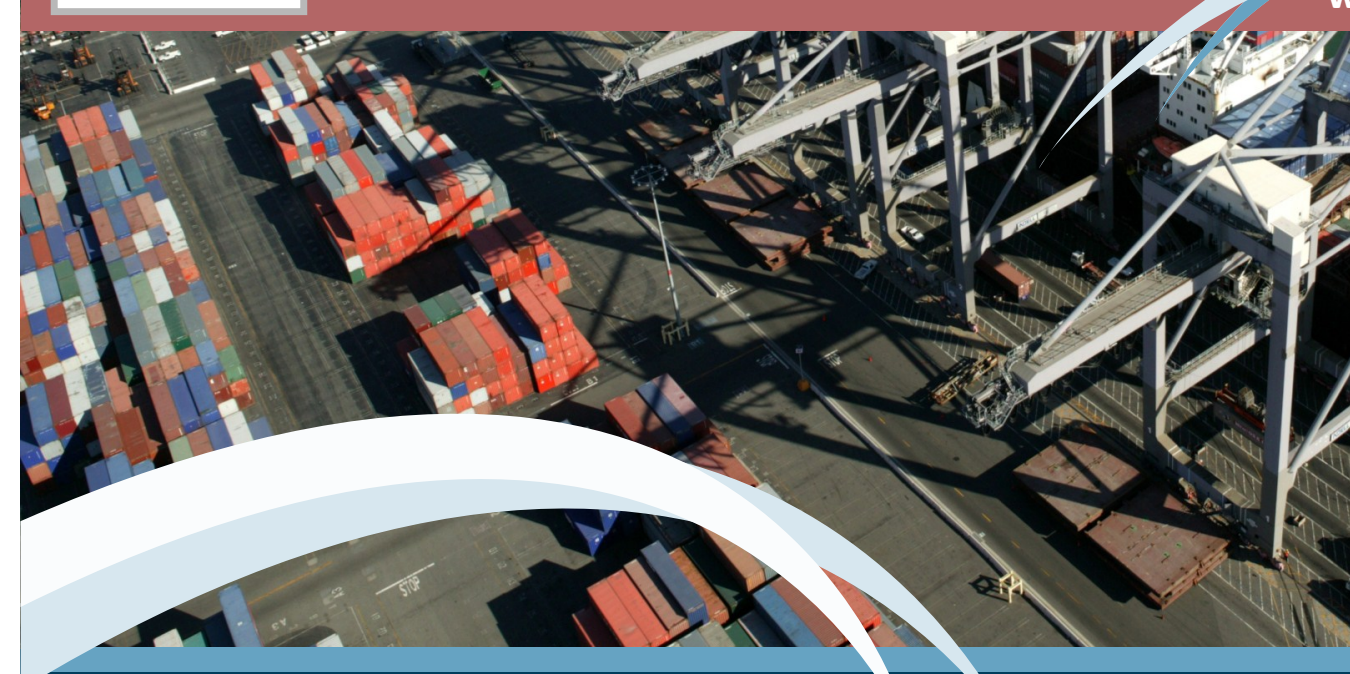

www.ams.usda.gov/oscar

\section{Weekly Container Overview}

Container Availability by Location:

- Ports: The ports of Los Angeles/Long Beach, New York, Norfolk, and Charleston are expected to have the most available containers among the reported port locations over the next 3 weeks. The container availability estimates have increased over the previous week for all these ports except New York. The ports of New Orleans, Seattle, and Oakland also experienced significant increases over the previous week.

- Inland Locations: Among the reported inland locations, Chicago, Dallas, Memphis, and Columbus are expected to have the most containers available over the next 3 weeks. Except for Dallas and Columbus, each of these locations saw increases over the previous week's estimates. In fact, over the past several weeks, Dallas and Minneapolis have experienced consistent container availability increases.

- Equipment: Overall, availability for all container types combined was up more than 3,300 containers this week over the previous week.

- More than half of the locations saw an increase in overall availability, but availability of refrigerated containers at most locations fell from the previous week.

- The largest availability jump this week was in New Orleans, mostly for $20 \mathrm{ft}$ and $40 \mathrm{ft}$ dry containers.

- Among the 5 equipment types reported, the largest increase this week was for $40 \mathrm{ft}$ dry containers, particularly in Memphis, New Orleans, Houston, Minneapolis, and Charleston.

- Over the next 3 weeks, Cincinnati shows a sizeable increase in availability of $40 \mathrm{ft}$ high-cube containers.

January 23, 2013

Understanding this Report P.2

National Overview Maps P. 3-5

Charleston, SC P.6

Chicago, IL P.7

Cincinnati, OH P.8

Columbus, OH P.9

Dallas, TX P.10

Denver, CO P.11

Houston, TX P.12

Kansas City, MO P.13

Los Angeles and Long Beach, CA P.14

Memphis, TN P.15

Minneapolis, MN P.16

New Orleans, LA P.17

New York, NY P.18

Norfolk, VA P.19

Oakland, CA P.20

Savannah, GA P.21

Seattle, WA P.22

Tacoma, WA P.23

To subscribe to OSCAR, e-mail: April.Taylor@ams.usda.gov 


\section{Understanding this Report}

The weekly Ocean Shipping Container Availability Report (OCSAR) provides a snapshot of container availability for westbound transpacific trade lanes at select intermodal locations around the country. The data presented in this report are gathered from the 8 member carriers in the Westbound Transpacific Stabilization Agreement (WTSA). The WTSA carriers include COSCO, Evergreen, Hanjin Shipping, Hapag Lloyd, Yang Ming Transport Corporation, OOCL, K Line, and Hyundai Merchant Marine.

- The data are estimates of container availability per week by the participating carriers. The carriers determine container availability by comparing current supplies at each of the 18 intermodal locations with the demand for these containers based on up-to-date bookings or reservation information in the westbound transpacific trade lane.

- $\quad$ Each week, participating carriers report the estimated number of containers available for the current week (W1) as well as estimates for the subsequent 2 weeks (W2 and W3).

- Maps on pages 3-5 offer a national overview of weekly container availability, as well as an average of availability over the past 6 months. The weekly national data are derived from the sum of the individual carrier responses at each intermodal location.

- Individual carrier responses are presented by location on pages 6-23 and have been sorted from largest to smallest. To maintain carrier confidentiality, specific carrier identification is not provided.

- Carrier responses showing negative numbers, such as -29 , should be interpreted as meaning that 29 containers are needed by shippers but the carrier is not able to supply them. A positive number, such as 64 , would mean 64 containers are available that are not demanded. An indication of 0 means the carriers have the exact amount of containers needed based on current supply and demand estimates.

- In addition to the weekly container availability data, the participating carriers provide average weekly throughput data for the past 6 months. These data help readers understand the aggregate flow of containers that have moved through the 18 select locations by the 8 participating carriers over the previous 6 months. These data will only be updated every 6 months.

- Availability levels are subject to change, and may be affected by delays in return of equipment to carriers by shippers or consignees, and by free time restrictions imposed by railroads. Due to the dynamic nature of the container industry, carriers cannot be held responsible for the level of availability at the time of inquiry.

- $\quad$ Data presented in the report do not imply availability of chassis.

- Container availability estimates represent availability for all export cargo, not just agricultural products.

- USDA collects these data on a voluntary basis and cannot be held responsible for the status of container availability when a reader makes inquiry with an ocean carrier. USDA relies on the participating ocean carriers to ensure the data is accurate.

- Terminology:

20ft Dry Container (20ft): A standard shipping container that is 20 feet long.

40ft Dry Container (40ft): A standard shipping container that is 40 feet long.

40ft High-Cube (HC): A shipping container which is 40 feet long and 9 feet, 6 inches tall (1 foot taller than a standard $40 f t$ dry container).

40ft reefer: A refrigerated shipping container which is 40 feet long.

20ft reefer: A refrigerated shipping container which is 20 feet long.

Chassis: A wheeled frame to which a shipping container can be locked for storage or movement. (Plural chassis) 


\section{National Overview}

\section{Week of Jan 23-29, 2013}

\section{Six-Month Snapshot of Average Weekly Throughput}

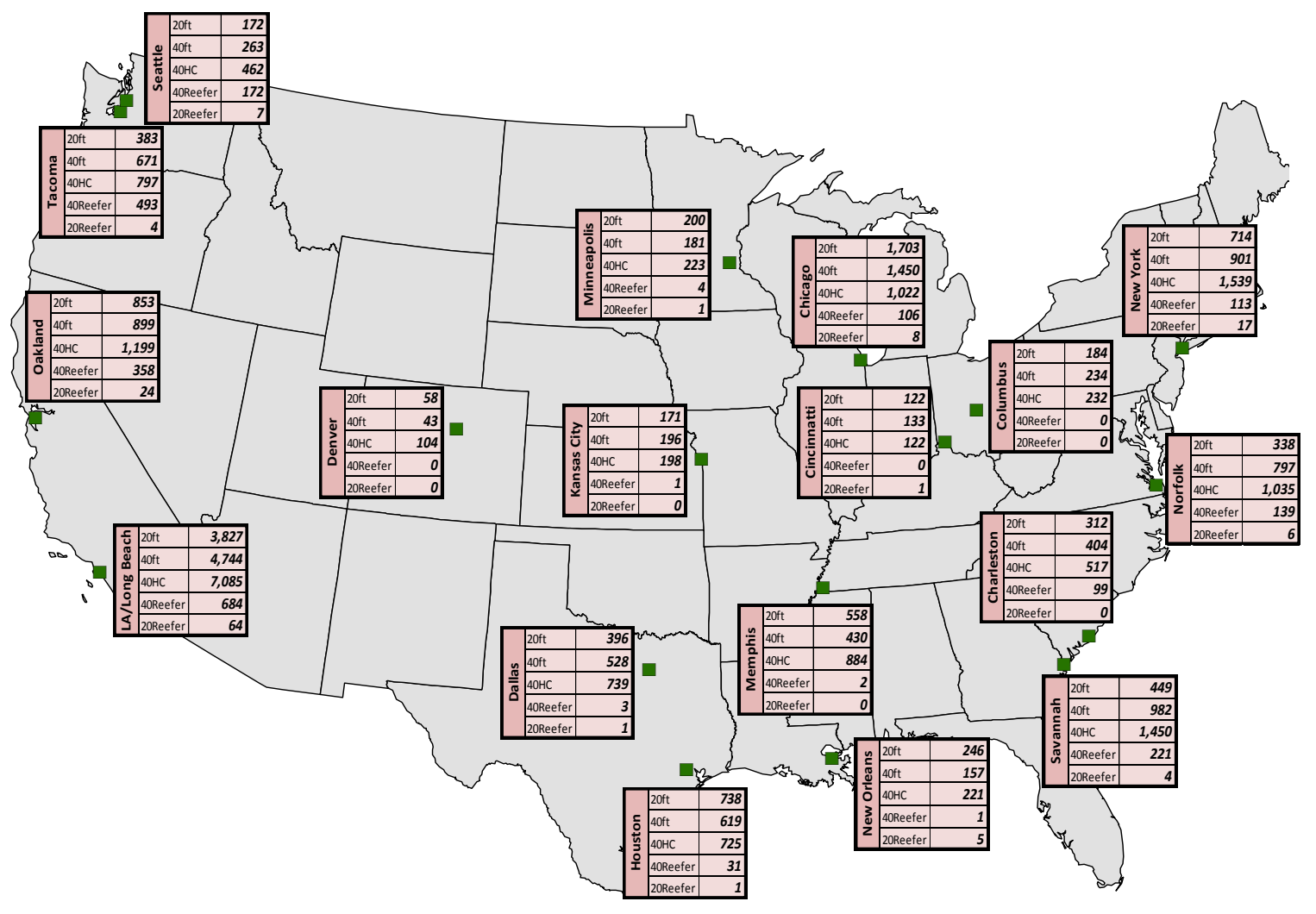

\section{Average Weekly Availability for 20ft Dry Containers}

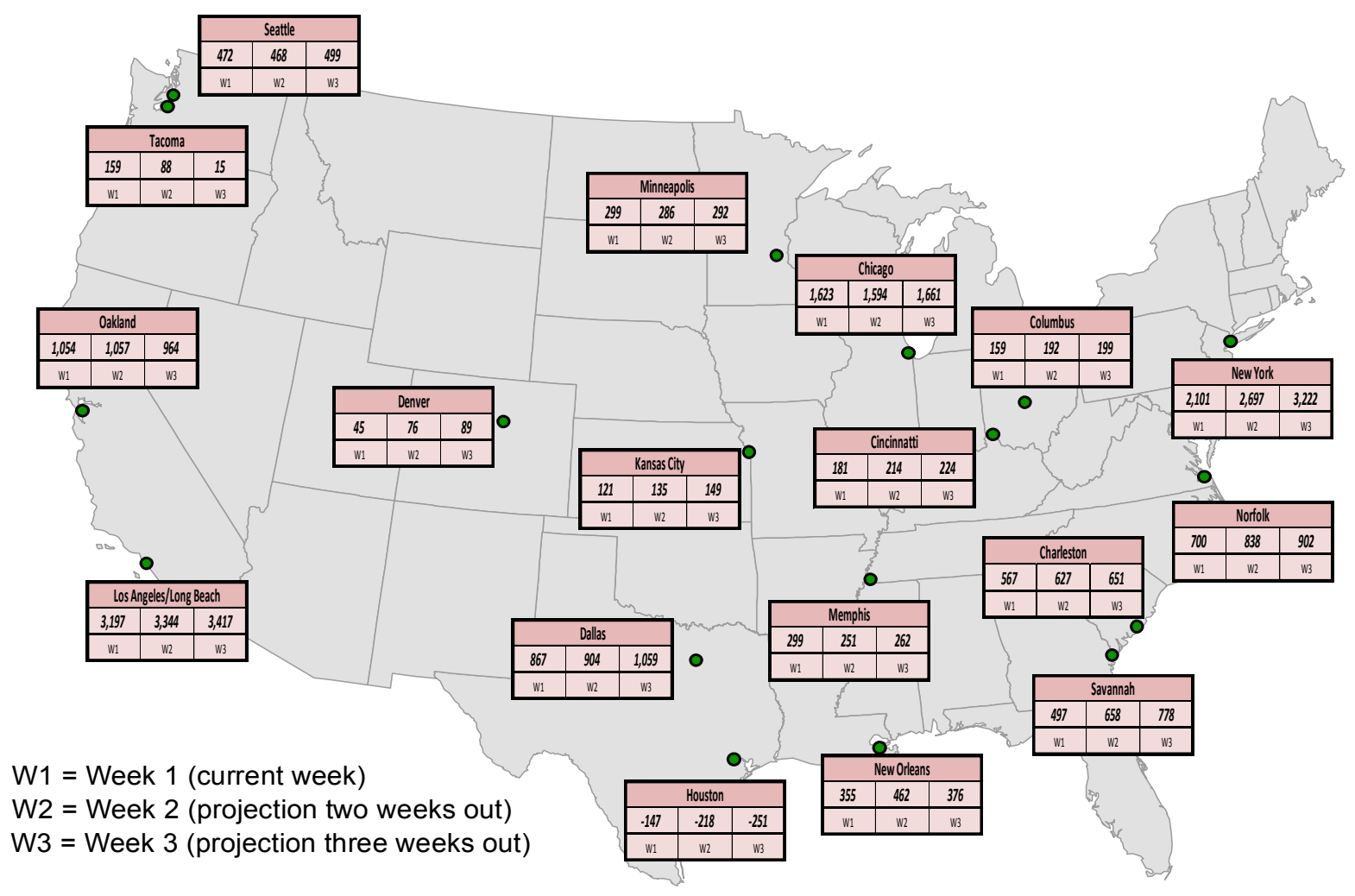




\section{National Overview}

\section{Week of Jan 23-29, 2013}

\section{Average Weekly Availability for 40ft Dry Containers}

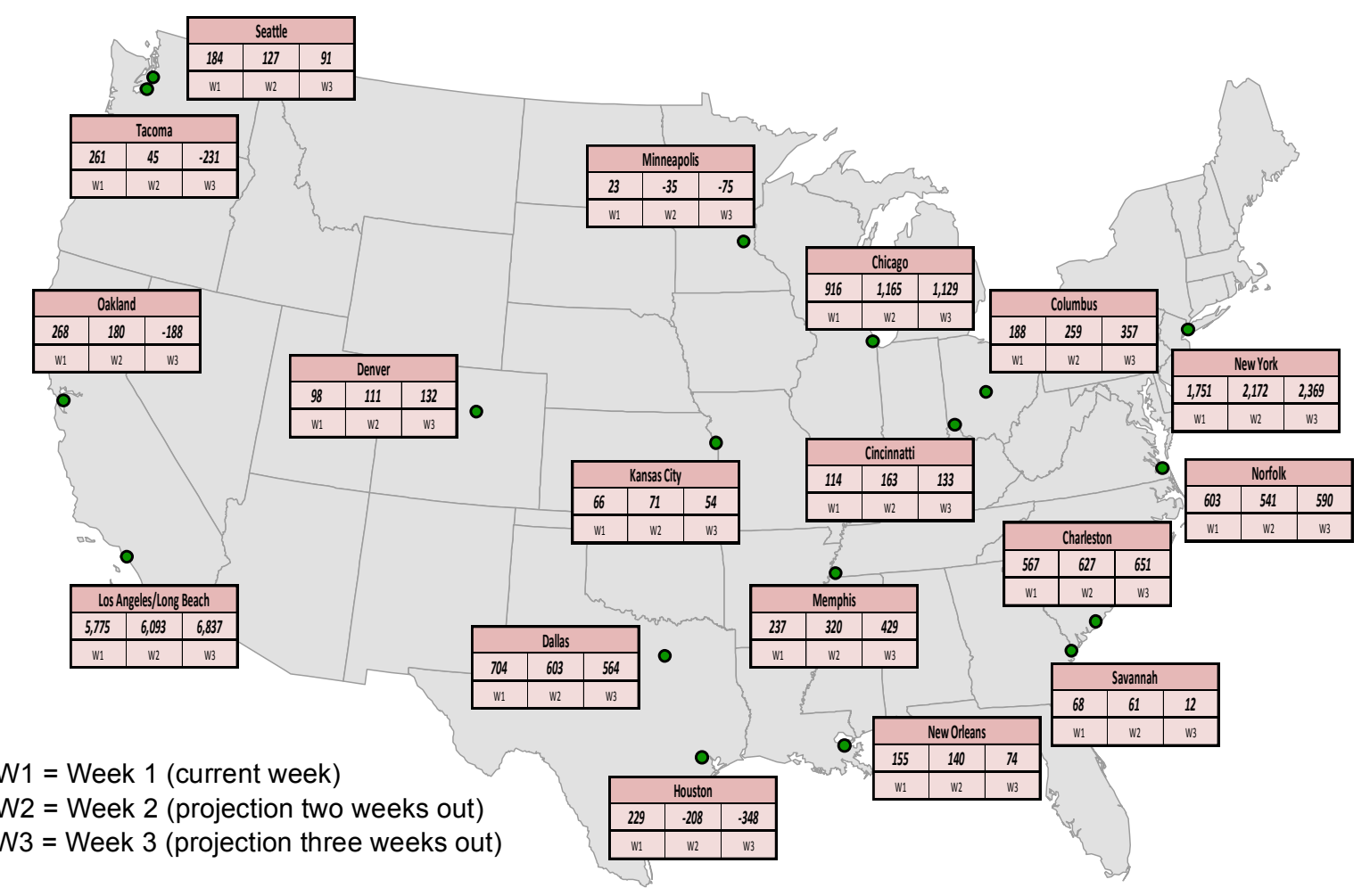

\section{Average Weekly Availability for $40 \mathrm{ft}$ High Cube Containers}

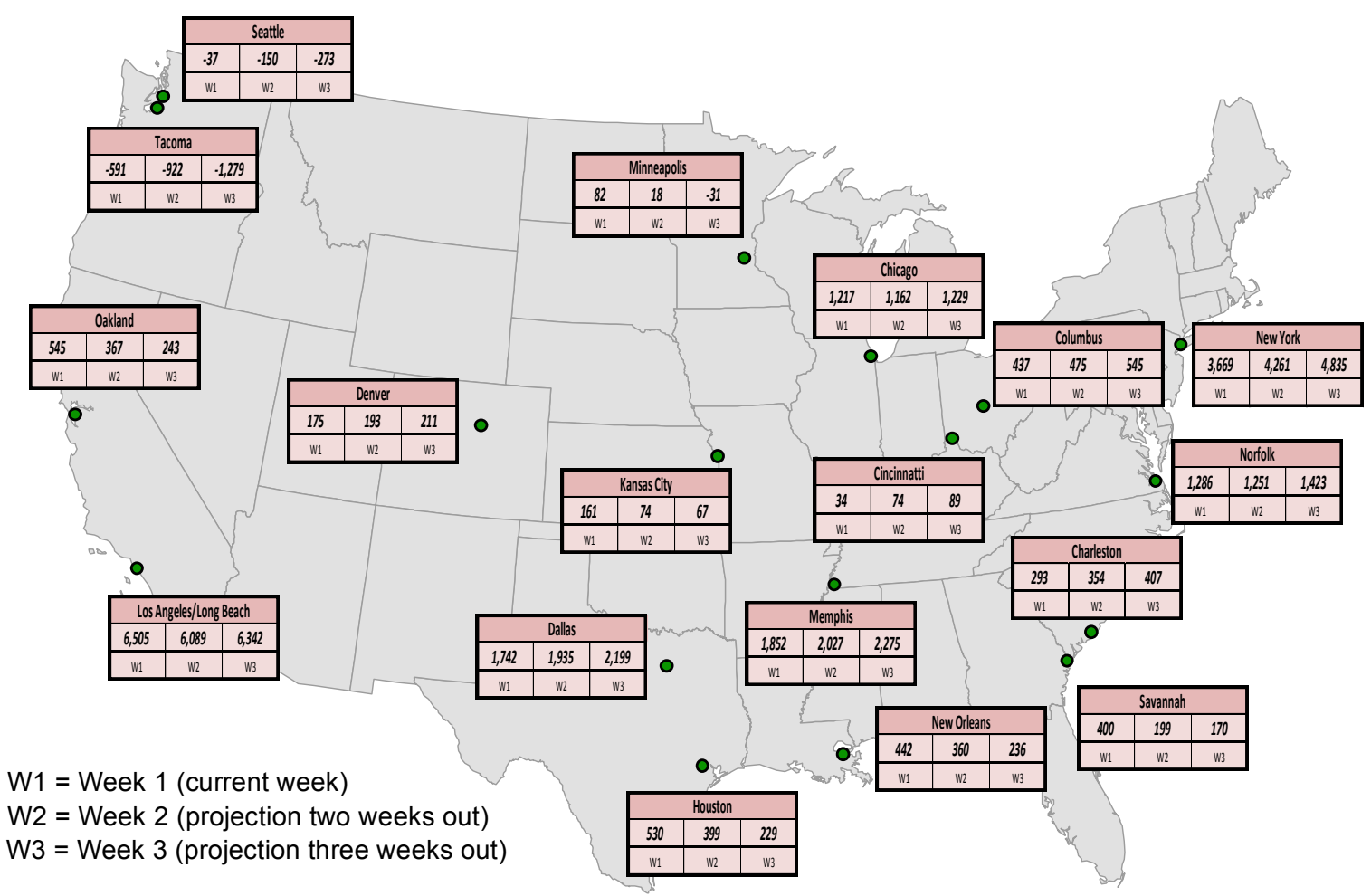




\section{National Overview}

\section{Week of Jan 23-29, 2013}

Average Weekly Availability for 40ft Refrigerated Containers

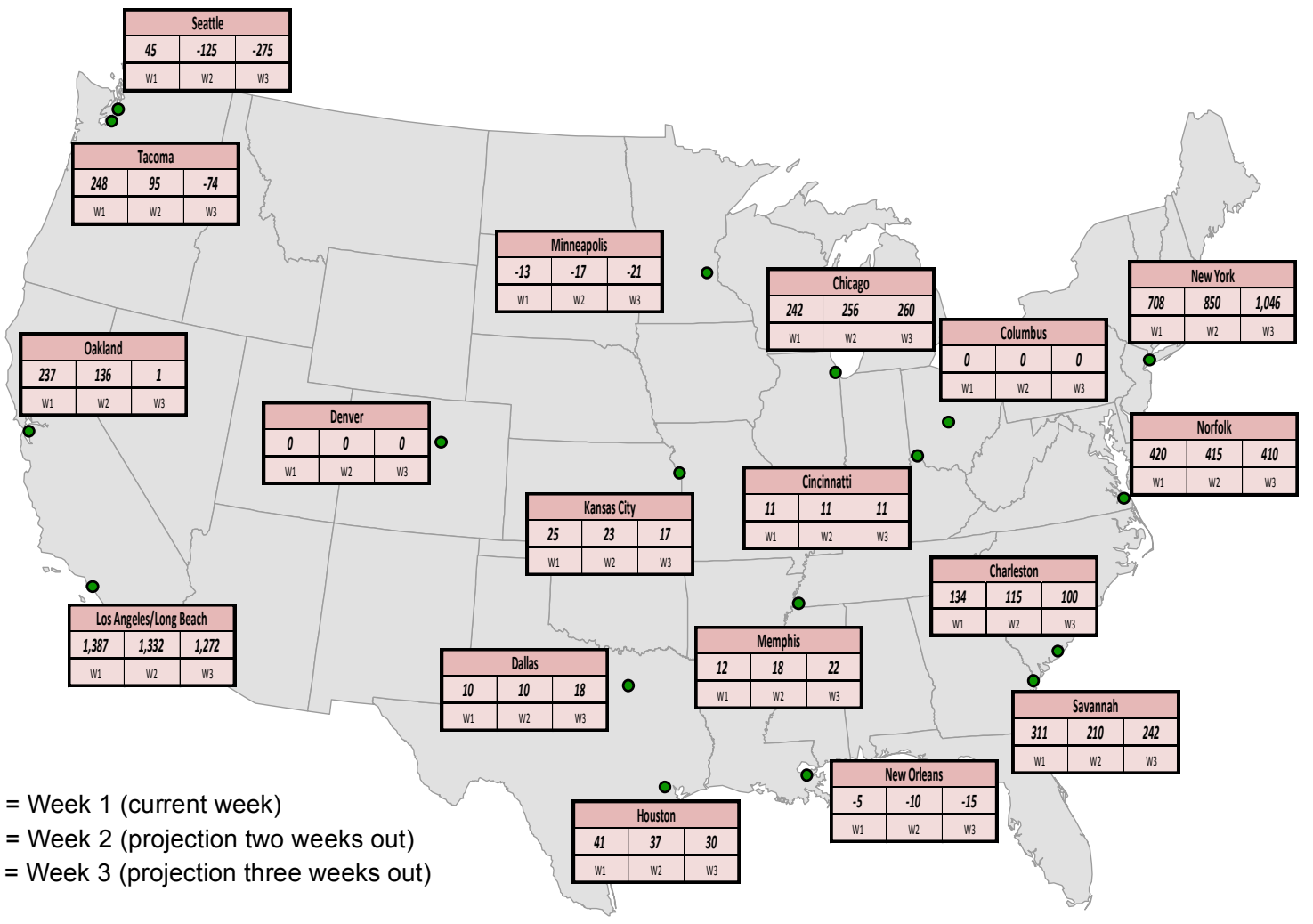

Average Weekly Availability for $20 \mathrm{ft}$ Refrigerated Containers

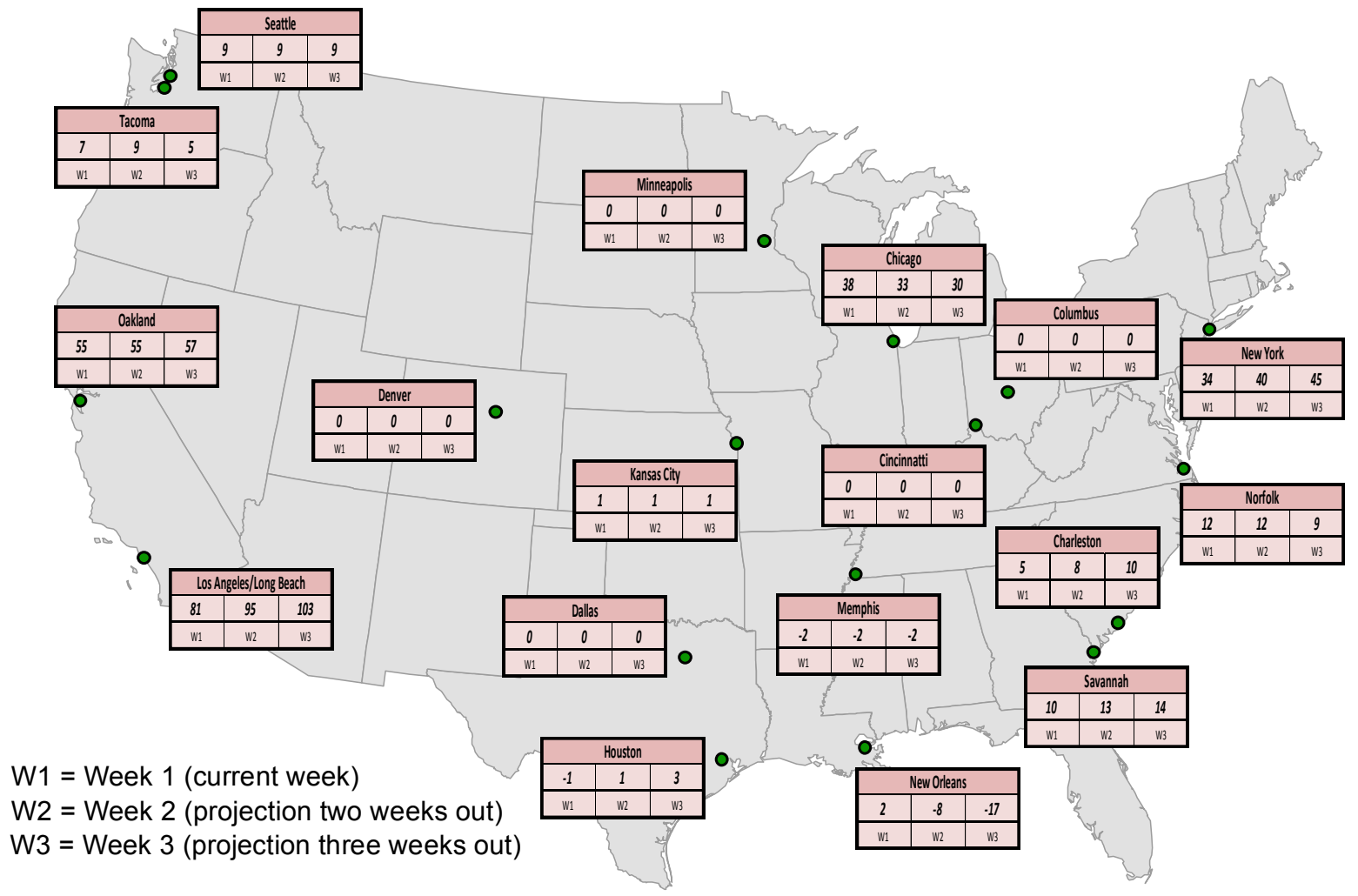




\section{Charleston, SC}

\begin{tabular}{|c|c|c|c|c|c|c|c|c|c|c|}
\hline \multicolumn{11}{|c|}{ Charleston } \\
\hline \multicolumn{11}{|c|}{ 20ft Dry } \\
\hline & \multicolumn{9}{|c|}{ Carrier Availability Responses } & Total \\
\hline Current Week: Jan 23-29 & 166 & 150 & 104 & 80 & 70 & 5 & 3 & -11 & . & 567 \\
\hline Week 2: Jan 30-Feb 5 & 127 & 150 & 160 & 70 & 70 & 8 & 25 & 17 & . & 627 \\
\hline Week 3: Feb 6-12 & 115 & 150 & 187 & 72 & 70 & 7 & 23 & 27 & . & 651 \\
\hline \multicolumn{11}{|c|}{ 40ft Dry } \\
\hline & \multicolumn{9}{|c|}{ Carrier Availability Responses } & Total \\
\hline Current Week: Jan 23-29 & 286 & 222 & 100 & 72 & 26 & 25 & 8 & 4 & . & 743 \\
\hline Week 2: Jan 30-Feb 5 & 369 & 160 & 100 & 72 & 24 & 29 & 7 & 12 & . & 773 \\
\hline Week 3: Feb 6-12 & 437 & 130 & 100 & 72 & 23 & 18 & 8 & 19 & . & 807 \\
\hline \multicolumn{11}{|c|}{ 40ft High Cube } \\
\hline & \multicolumn{9}{|c|}{ Carrier Availability Category Responses } & Total \\
\hline Current Week: Jan 23-29 & 93 & 90 & 89 & 61 & 10 & 10 & -5 & -55 & . & 293 \\
\hline Week 2: Jan 30-Feb 5 & 60 & 160 & 99 & 61 & 10 & 10 & 12 & -58 & . & 354 \\
\hline Week 3: Feb 6-12 & 50 & 213 & 114 & 61 & 10 & 9 & 26 & -76 & . & 407 \\
\hline \multicolumn{11}{|c|}{ 40ft Refrigerated } \\
\hline & \multicolumn{9}{|c|}{ Carrier Availability Category Responses } & Total \\
\hline Current Week: Jan 23-29 & 120 & 20 & 15 & 5 & 0 & -1 & -25 & . & . & 134 \\
\hline Week 2: Jan 30-Feb 5 & 123 & 20 & 15 & 4 & 0 & 0 & -47 & . & . & 115 \\
\hline Week 3: Feb 6-12 & 112 & 19 & 15 & 4 & 0 & 1 & -51 & . & . & 100 \\
\hline \multicolumn{11}{|c|}{ 20ft Refrigerated } \\
\hline & \multicolumn{9}{|c|}{ Carrier Availability Category Responses } & Total \\
\hline Current Week: Jan 23-29 & 4 & 1 & 0 & . & 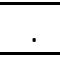 & . & . & . & . & 5 \\
\hline Week 2: Jan 30-Feb 5 & 6 & 1 & 1 & . & . &. & . & . & . & 8 \\
\hline Week 3: Feb 6-12 & 7 & 1 & 2 & . & 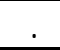 & . & . & & . & 10 \\
\hline
\end{tabular}

Note: "." denotes no response from carriers.

\section{Six-Month Snapshot of Average Weekly Throughput}

June 2012-November 2012

\begin{tabular}{|l|c|c|}
\hline Container Types & $\begin{array}{c}\text { Average Total } \\
\text { Weekly Throughput* }\end{array}$ & $\begin{array}{c}\text { Range of Individual } \\
\text { Carrier Responses }\end{array}$ \\
\hline 20ft & 312 & $0 \ldots 108$ \\
40ft & 404 & $0 \ldots 200$ \\
40ft High Cubes & 517 & $17 \ldots 200$ \\
40ft Refrigerated & 99 & $0 \ldots 60$ \\
20ft Refrigerated & 0 & $0 . .0$ \\
\hline
\end{tabular}

*Data reflect the sum of the individual carriers' average weekly throughput over a 6month timeframe 


\section{Chicago, IL}

\begin{tabular}{|c|c|c|c|c|c|c|c|c|c|c|c|}
\hline \multicolumn{12}{|c|}{$\frac{\text { Chicago }}{\text { 20ft Dry }}$} \\
\hline & \multicolumn{10}{|c|}{ Carrier Availability Category Responses } & Total \\
\hline Current Week: Jan 23-29 & 979 & 400 & 158 & 95 & 19 & 10 & -4 & -34 & . & . & 1,623 \\
\hline Week 2: Jan 30-Feb 5 & 1,144 & 400 & 41 & 95 & -148 & 10 & 48 & 4 & . & . & 1,594 \\
\hline Week 3: Feb 6-12 & 1,417 & 400 & -124 & 95 & -223 & 5 & 89 & 2 & . & . & 1,661 \\
\hline \multicolumn{12}{|c|}{ 40ft Dry } \\
\hline & \multicolumn{10}{|c|}{ Carrier Availability Category Responses } & Total \\
\hline Current Week: Jan 23-29 & 442 & 300 & 198 & 13 & 11 & 9 & -9 & -48 & . & . & 916 \\
\hline Week 2: Jan 30-Feb 5 & 537 & 300 & 124 & 13 & 11 & 105 & 78 & -3 & . & . & 1,165 \\
\hline Week 3: Feb 6-12 & 636 & 300 & 131 & 13 & 11 & -156 & 214 & -20 & . & . & 1,129 \\
\hline \multicolumn{12}{|c|}{ 40ft High Cube } \\
\hline & \multicolumn{10}{|c|}{ Carrier Availability Category Responses } & Total \\
\hline Current Week: Jan 23-29 & 600 & 349 & 189 & 79 & 25 & 13 & 12 & -50 & . & . & 1,217 \\
\hline Week 2: Jan 30-Feb 5 & 600 & 310 & 141 & 66 & 25 & 6 & 12 & 2 & . & $\cdot$ & 1,162 \\
\hline Week 3: Feb 6-12 & 600 & 246 & 268 & -35 & 25 & 4 & 12 & 109 & . & . & 1,229 \\
\hline \multicolumn{12}{|c|}{ 40ft Refrigerated } \\
\hline & \multicolumn{10}{|c|}{ Carrier Availability Category Responses } & Total \\
\hline Current Week: Jan 23-29 & 137 & 102 & 10 & 0 & 0 & -7 & . & . & . & . & 242 \\
\hline Week 2: Jan 30-Feb 5 & 161 & 92 & 10 & 0 & 0 & -7 & . & . & . & . & 256 \\
\hline Week 3: Feb 6-12 & 172 & 85 & 11 & 0 & -1 & -7 & . & . & . & $\cdot$ & 260 \\
\hline \multicolumn{12}{|c|}{ 20ft Refrigerated } \\
\hline & \multicolumn{10}{|c|}{ Carrier Availability Category Responses } & Total \\
\hline Current Week: Jan 23-29 & 23 & 15 & . & . & . & . & . & . & . & . & 38 \\
\hline Week 2: Jan 30-Feb 5 & 20 & 13 & . & . & . & . & . & . & . & . & 33 \\
\hline Week 3: Feb 6-12 & 17 & 13 & . & . & . & 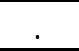 & . & . & . & . & 30 \\
\hline
\end{tabular}

Note: "." denotes no response from carriers.

\begin{tabular}{|l|c|c|}
\hline \multicolumn{3}{|c|}{ Six-Month Snapshot of Average Weekly } \\
Throughput \\
June 2012-November 2012 \\
\hline Container Types & Average Total & Range of Individual \\
& Weekly Throughput* & Carrier Responses \\
\hline 20ft & 1,703 & $93 \ldots 600$ \\
40ft & 1,450 & $5 \ldots 302$ \\
40ft High Cubes & 1,022 & $34 . .405$ \\
40ft Refrigerated & 106 & $0 . .51$ \\
20ft Refrigerated & 8 & $0 . . .4$ \\
\hline
\end{tabular}

*Data reflect the sum of the individual carriers' average weekly throughput over a 6month timeframe 


\section{Cincinnati, $\mathrm{OH}$}

\begin{tabular}{|c|c|c|c|c|c|c|c|c|c|c|c|}
\hline \multicolumn{12}{|c|}{ Cincinnatti } \\
\hline \multicolumn{12}{|c|}{ 20ft Dry } \\
\hline & \multicolumn{10}{|c|}{ Carrier Availability Category Responses } & Total \\
\hline Current Week: Jan 23-29 & 56 & 36 & 23 & 23 & 15 & 13 & 10 & 5 & . & . & 181 \\
\hline Week 2: Jan 30-Feb 5 & 76 & 49 & 23 & 27 & 14 & 15 & 10 & 0 & . & . & 214 \\
\hline Week 3: Feb 6-12 & 55 & 64 & 23 & 23 & 19 & 29 & 10 & 1 & . & . & 224 \\
\hline \multicolumn{12}{|c|}{ 40ft Dry } \\
\hline & \multicolumn{10}{|c|}{ Carrier Availability Category Responses } & Total \\
\hline Current Week: Jan 23-29 & 30 & 24 & 22 & 15 & 10 & 7 & 7 & -1 & . & . & 114 \\
\hline Week 2: Jan 30-Feb 5 & 45 & 50 & 39 & 15 & 10 & 2 & 3 & -1 & . & . & 163 \\
\hline Week 3: Feb 6-12 & 26 & 22 & 60 & 17 & 10 & -3 & 2 & -1 & . & . & 133 \\
\hline \multicolumn{12}{|c|}{ 40ft High Cube } \\
\hline & \multicolumn{10}{|c|}{ Carrier Availability Category Responses } & Total \\
\hline Current Week: Jan 23-29 & 36 & 35 & 19 & 10 & 2 & -7 & -11 & -50 & . & . & 34 \\
\hline Week 2: Jan 30-Feb 5 & 31 & 39 & 15 & 19 & 32 & -7 & -5 & -50 & . & . & 74 \\
\hline Week 3: Feb 6-12 & 26 & 51 & 12 & 33 & 31 & -7 & -7 & -50 & . & . & 89 \\
\hline \multicolumn{12}{|c|}{ 40ft Refrigerated } \\
\hline & \multicolumn{10}{|c|}{ Carrier Availability Category Responses } & Total \\
\hline Current Week: Jan 23-29 & 11 & 0 & . & . & . & . & . & . & . & . & 11 \\
\hline Week 2: Jan 30-Feb 5 & 11 & 0 & . & . & . & . & . & . & . & . & 11 \\
\hline Week 3: Feb 6-12 & 11 & 0 & . & . & . & . & . & . & . & . & 11 \\
\hline \multicolumn{12}{|c|}{ 20ft Refrigerated } \\
\hline & \multicolumn{10}{|c|}{ Carrier Availability Category Responses } & Total \\
\hline Current Week: Jan 23-29 & . & . & . & . & . & . & . & . & . & . & 0 \\
\hline Week 2: Jan 30-Feb 5 & . & . & . & . & . & . & . & . & . & . & 0 \\
\hline Week 3: Feb 6-12 & . & . & . & . & . & . & . & . & . & . & 0 \\
\hline
\end{tabular}

Note: "." denotes no response from carriers.

\begin{tabular}{|l|c|c|}
\hline \multicolumn{3}{|c|}{ Six-Month Snapshot of Average Weekly } \\
Throughput \\
June 2012-November 2012 \\
\hline Container Types & $\begin{array}{c}\text { Average Total } \\
\text { Weekly Throughput* }\end{array}$ & $\begin{array}{c}\text { Range of Individual } \\
\text { Carrier Responses }\end{array}$ \\
\hline 20ft & 122 & $7 \ldots 35$ \\
40ft & 133 & $8 \ldots 30$ \\
40ft High Cubes & 122 & $11 \ldots 35$ \\
40ft Refrigerated & 0 & $0 \ldots 0$ \\
20ft Refrigerated & 1 & $0 \ldots 1$ \\
\hline
\end{tabular}

*Data reflect the sum of the individual carriers' average weekly throughput over a 6month timeframe 


\section{Columbus, $\mathrm{OH}$}

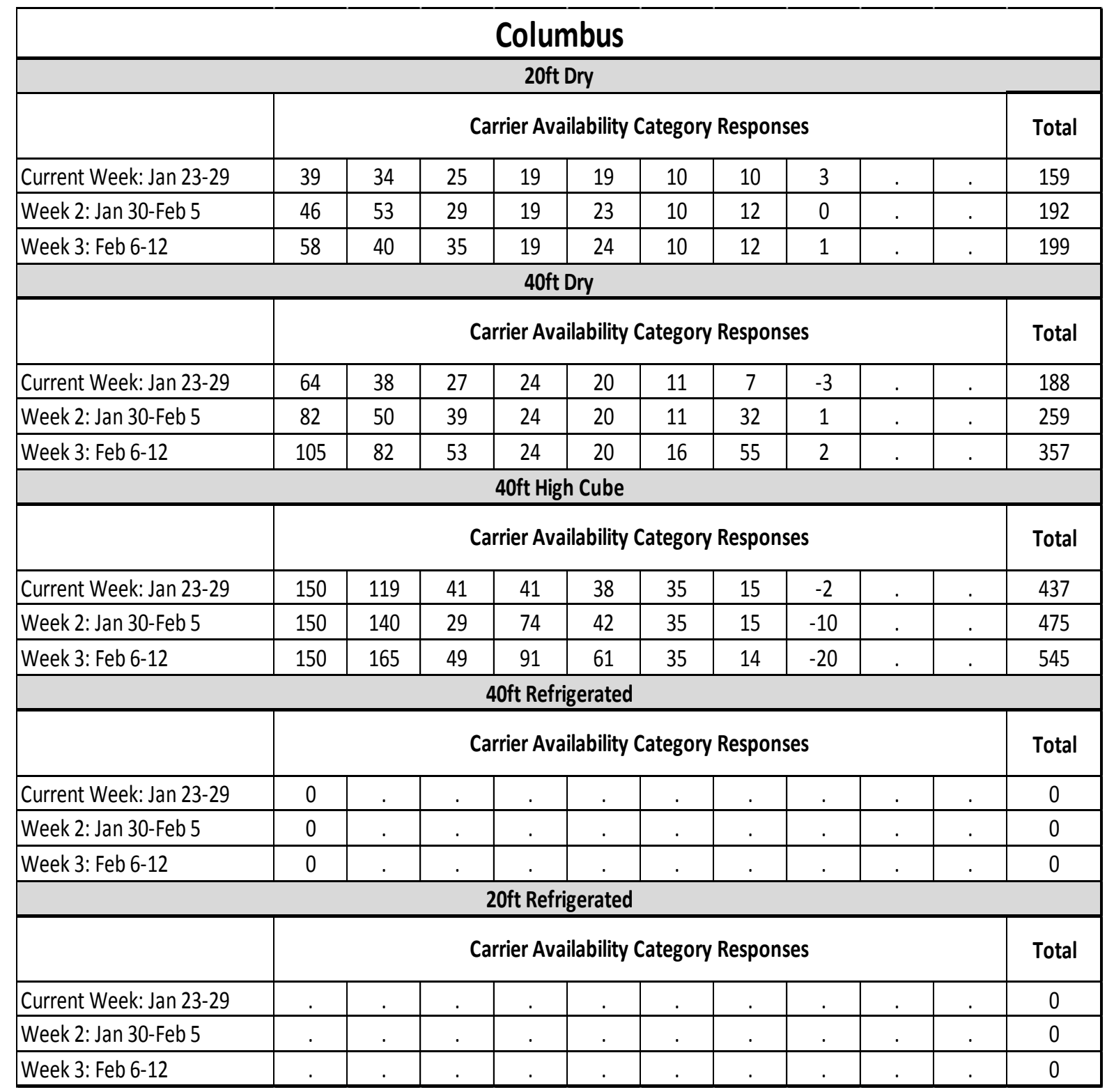

Note: "." denotes no response from carriers.

\begin{tabular}{|l|c|c|}
\hline \multicolumn{3}{|c|}{ Six-Month Snapshot of Average Weekly } \\
Throughput \\
June 2012-November 2012 \\
\hline Container Types & $\begin{array}{c}\text { Average Total } \\
\text { Weekly Throughput* }\end{array}$ & $\begin{array}{c}\text { Range of Individual } \\
\text { Carrier Responses }\end{array}$ \\
\hline $\mathbf{2 0 f t}$ & 184 & $8 \ldots 60$ \\
$\mathbf{4 0 f t}$ & 234 & $6 \ldots 80$ \\
$\mathbf{4 0 f t}$ High Cubes & 232 & $15 \ldots 60$ \\
$\mathbf{4 0 f t}$ Refrigerated & 0 & $0 \ldots 0$ \\
20ft Refrigerated & 0 & $0 \ldots 0$ \\
\hline
\end{tabular}

*Data reflect the sum of the individual carriers' average weekly throughput over a 6month timeframe 


\section{Dallas, TX}

\section{Dallas}

\begin{tabular}{|c|c|c|c|c|c|c|c|c|c|c|}
\hline \multicolumn{11}{|c|}{ Dallas } \\
\hline \multicolumn{11}{|c|}{ 20ft Dry } \\
\hline & \multicolumn{9}{|c|}{ Carrier Availability Category Responses } & \multirow{2}{*}{$\begin{array}{c}\text { Total } \\
867\end{array}$} \\
\hline Current Week: Jan 23-29 & 203 & 193 & 160 & 119 & 85 & 75 & 20 & 12 & . & \\
\hline Week 2: Jan 30-Feb 5 & 294 & 198 & 116 & 48 & 136 & 75 & 25 & 12 & . & 904 \\
\hline Week 3: Feb 6-12 & 396 & 278 & 105 & 0 & 168 & 75 & 25 & 12 & . & 1,059 \\
\hline \multicolumn{11}{|c|}{ 40ft Dry } \\
\hline & \multicolumn{9}{|c|}{ Carrier Availability Category Responses } & Total \\
\hline Current Week: Jan 23-29 & 175 & 173 & 171 & 128 & 22 & 20 & 16 & -1 & . & 704 \\
\hline Week 2: Jan 30-Feb 5 & 268 & 56 & 130 & 58 & 19 & 20 & 53 & -1 & . & 603 \\
\hline Week 3: Feb 6-12 & 377 & -2 & 38 & 50 & 25 & 20 & 57 & -1 & . & 564 \\
\hline \multicolumn{11}{|c|}{ 40ft High Cube } \\
\hline & \multicolumn{9}{|c|}{ Carrier Availability Category Responses } & Total \\
\hline Current Week: Jan 23-29 & 620 & 330 & 214 & 161 & 160 & 152 & 83 & 22 & . & 1,742 \\
\hline Week 2: Jan 30-Feb 5 & 775 & 420 & 131 & 276 & 160 & 70 & 83 & 20 & . & 1,935 \\
\hline Week 3: Feb 6-12 & 948 & 568 & 125 & 300 & 160 & -10 & 83 & 25 & . & 2,199 \\
\hline \multicolumn{11}{|c|}{ 40ft Refrigerated } \\
\hline & \multicolumn{9}{|c|}{ Carrier Availability Category Responses } & Total \\
\hline Current Week: Jan 23-29 & 21 & 0 & -11 & . & . & . & . & . & . & 10 \\
\hline Week 2: Jan 30-Feb 5 & 23 & 0 & -13 & . & . & . & . & . & . & 10 \\
\hline Week 3: Feb 6-12 & 33 & 0 & -15 & . & . & . & . &. & . & 18 \\
\hline \multicolumn{11}{|c|}{ 20ft Refrigerated } \\
\hline & \multicolumn{9}{|c|}{ Carrier Availability Category Responses } & Total \\
\hline Current Week: Jan 23-29 & 0 & - & . & . & . & $\cdot$ & . & . & . & 0 \\
\hline Week 2: Jan 30-Feb 5 & 0 & . & . & . & . & . & . & . & . & 0 \\
\hline Week 3: Feb 6-12 & 0 & . & . & . & . & . &. &. & . & 0 \\
\hline
\end{tabular}

Note: "." denotes no response from carriers.

\begin{tabular}{|l|c|c|}
\hline \multicolumn{3}{|c|}{ Six-Month Snapshot of Average Weekly } \\
Throughput \\
June 2012-November 2012 \\
\hline Container Types & $\begin{array}{c}\text { Average Total } \\
\text { Weekly Throughput* }\end{array}$ & $\begin{array}{c}\text { Range of Individual } \\
\text { Carrier Responses }\end{array}$ \\
\hline $\mathbf{2 0 f t}$ & 396 & $5 \ldots 150$ \\
$\mathbf{4 0 f t}$ & 528 & $12 \ldots .250$ \\
$\mathbf{4 0 f t ~ H i g h ~ C u b e s ~}$ & 739 & $22 \ldots .250$ \\
$\mathbf{4 0 f t ~ R e f r i g e r a t e d ~}$ & 3 & $0 . .3$ \\
$\mathbf{2 0 f t}$ Refrigerated & 1 & $0 . .1$ \\
\hline
\end{tabular}

*Data reflect the sum of the individual carriers' average weekly throughput over a 6month timeframe 


\section{Denver, CO}

\begin{tabular}{|c|c|c|c|c|c|c|c|c|c|c|c|}
\hline \multicolumn{12}{|c|}{ Denver } \\
\hline \multicolumn{12}{|c|}{ 20ft Dry } \\
\hline & \multicolumn{10}{|c|}{ Carrier Availability Category Responses } & Total \\
\hline Current Week: Jan 23-29 & 44 & 20 & 11 & 8 & 4 & -7 & -15 & -20 & . & . & 45 \\
\hline Week 2: Jan 30-Feb 5 & 64 & 20 & 15 & -1 & 5 & -7 & -9 & -11 & . & . & 76 \\
\hline Week 3: Feb 6-12 & 83 & 20 & 19 & -5 & 5 & -7 & -14 & -12 & . & . & 89 \\
\hline \multicolumn{12}{|c|}{ 40ft Dry } \\
\hline & \multicolumn{10}{|c|}{ Carrier Availability Category Responses } & Total \\
\hline Current Week: Jan 23-29 & 29 & 23 & 15 & 11 & 10 & 6 & 5 & -1 & . & . & 98 \\
\hline Week 2: Jan 30-Feb 5 & 26 & 34 & 8 & 16 & 10 & 12 & 5 & 0 & . & . & 111 \\
\hline Week 3: Feb 6-12 & 29 & 43 & 6 & 15 & 10 & 28 & 5 & -4 & . & . & 132 \\
\hline \multicolumn{12}{|c|}{ 40ft High Cube } \\
\hline & \multicolumn{10}{|c|}{ Carrier Availability Category Responses } & Total \\
\hline Current Week: Jan 23-29 & 77 & 40 & 17 & 14 & 11 & 10 & 7 & -1 & . & . & 175 \\
\hline Week 2: Jan 30-Feb 5 & 99 & 40 & 21 & 14 & 3 & 10 & 3 & 3 & . & . & 193 \\
\hline Week 3: Feb 6-12 & 117 & 40 & 25 & 14 & 0 & 10 & 2 & 3 & . & . & 211 \\
\hline \multicolumn{12}{|c|}{ 40ft Refrigerated } \\
\hline & \multicolumn{10}{|c|}{ Carrier Availability Category Responses } & Total \\
\hline Current Week: Jan 23-29 & 0 & 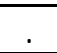 & & . & . & . & . & . & . & . & 0 \\
\hline Week 2: Jan 30-Feb 5 & 0 & . & &. & . & . & . &. & . & . & 0 \\
\hline Week 3: Feb 6-12 & 0 & . & . &. & . & . & . &. & . & . & 0 \\
\hline \multicolumn{12}{|c|}{ 20ft Refrigerated } \\
\hline & \multicolumn{10}{|c|}{ Carrier Availability Category Responses } & Total \\
\hline Current Week: Jan 23-29 & . & - & & . & . & 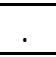 & . & . & . & . & 0 \\
\hline Week 2: Jan 30-Feb 5 & . & . & & . & . & . & . & . & . & . & 0 \\
\hline Week 3: Feb 6-12 & . & . & . & . & . & . & . & . & . & . & 0 \\
\hline
\end{tabular}

Note: "." denotes no response from carriers.

\section{Six-Month Snapshot of Average Weekly}

Throughput

June 2012-November 2012

\begin{tabular}{|l|c|c|}
\hline Container Types & $\begin{array}{c}\text { Average Total } \\
\text { Weekly Throughput* }\end{array}$ & $\begin{array}{c}\text { Range of Individual } \\
\text { Carrier Responses }\end{array}$ \\
\hline 20ft & 58 & $3 \ldots 17$ \\
40ft & 43 & $1 \ldots 10$ \\
40ft High Cubes & 104 & $1 \ldots . .50$ \\
40ft Refrigerated & 0 & $0 . . .0$ \\
20ft Refrigerated & 0 & $0 . . .0$ \\
\hline
\end{tabular}

*Data reflect the sum of the individual carriers' average weekly throughput over a 6month timeframe 


\section{Houston, TX}

\begin{tabular}{|c|c|c|c|c|c|c|c|c|c|c|c|}
\hline \multicolumn{12}{|c|}{ Houston } \\
\hline \multicolumn{12}{|c|}{ 20ft Dry } \\
\hline & \multicolumn{10}{|c|}{ Carrier Availability Category Responses } & Total \\
\hline Current Week: Jan 23-29 & 80 & 67 & 38 & 8 & -10 & -13 & -38 & -279 & . & . & -147 \\
\hline Week 2: Jan 30-Feb 5 & 80 & 18 & 31 & 9 & -10 & 26 & -9 & -363 & . & . & -218 \\
\hline Week 3: Feb 6-12 & 80 & 44 & 15 & 9 & -10 & 20 & 62 & -471 & . & . & -251 \\
\hline \multicolumn{12}{|c|}{ 40ft Dry } \\
\hline & \multicolumn{10}{|c|}{ Carrier Availability Category Responses } & Total \\
\hline Current Week: Jan 23-29 & 175 & 77 & 48 & 36 & 5 & 1 & -26 & -87 & . & . & 229 \\
\hline Week 2: Jan 30-Feb 5 & 175 & -16 & -45 & -9 & 5 & 2 & -84 & -236 & . & . & -208 \\
\hline Week 3: Feb 6-12 & 175 & -30 & -2 & -7 & 5 & 4 & -78 & -415 & . & . & -348 \\
\hline \multicolumn{12}{|c|}{ 40ft High Cube } \\
\hline & \multicolumn{10}{|c|}{ Carrier Availability Category Responses } & Total \\
\hline Current Week: Jan 23-29 & 293 & 175 & 161 & 8 & 5 & -11 & -24 & -77 & . & . & 530 \\
\hline Week 2: Jan 30-Feb 5 & 168 & 175 & 184 & -23 & 2 & -7 & -23 & -77 & . & . & 399 \\
\hline Week 3: Feb 6-12 & -3 & 175 & 250 & -30 & 1 & -19 & -68 & -77 & . & . & 229 \\
\hline \multicolumn{12}{|c|}{ 40ft Refrigerated } \\
\hline & \multicolumn{10}{|c|}{ Carrier Availability Category Responses } & Total \\
\hline \begin{tabular}{|l|} 
Current Week: Jan 23-29 \\
\end{tabular} & 30 & 5 & 4 & 2 & 0 & . & . & . & . & . & 41 \\
\hline Week 2: Jan 30-Feb 5 & 20 & 2 & 13 & 2 & 0 & . & . & . & . & . & 37 \\
\hline Week 3: Feb 6-12 & 10 & 0 & 18 & 2 & 0 & . & . & . & . & . & 30 \\
\hline \multicolumn{12}{|c|}{ 20ft Refrigerated } \\
\hline & \multicolumn{10}{|c|}{ Carrier Availability Category Responses } & Total \\
\hline Current Week: Jan 23-29 & 6 & 0 & -7 & & . & . & . & . & . & . & -1 \\
\hline Week 2: Jan 30-Feb 5 & 7 & 0 & -6 & . & . & . & 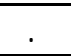 & . & . & . & 1 \\
\hline Week 3: Feb 6-12 & 8 & 0 & -5 & . & . & . & . & . & . & . & 3 \\
\hline
\end{tabular}

Note: "." denotes no response from carriers.

\begin{tabular}{|l|c|c|}
\hline \multicolumn{3}{|c|}{ Six-Month Snapshot of Average Weekly } \\
Throughput \\
June 2012-November 2012 \\
\hline Container Types & $\begin{array}{c}\text { Average Total } \\
\text { Weekly Throughput* }\end{array}$ & $\begin{array}{c}\text { Range of Individual } \\
\text { Carrier Responses }\end{array}$ \\
\hline 20ft & 738 & $50 \ldots 316$ \\
40ft & 619 & $50 \ldots 126$ \\
40ft High Cubes & 725 & $26 \ldots 355$ \\
40ft Refrigerated & 31 & $0 . .12$ \\
20ft Refrigerated & 1 & $0 . .1$ \\
\hline
\end{tabular}

*Data reflect the sum of the individual carriers' average weekly throughput over a 6month timeframe 


\section{Kansas City, MO}

\begin{tabular}{|c|c|c|c|c|c|c|c|c|c|c|c|}
\hline \multicolumn{12}{|c|}{ Kansas City } \\
\hline \multicolumn{12}{|c|}{ 20ft Dry } \\
\hline & \multicolumn{10}{|c|}{ Carrier Availability Category Responses } & Total \\
\hline Current Week: Jan 23-29 & 104 & 37 & 10 & 8 & 5 & 0 & -20 & -23 & . & . & 121 \\
\hline Week 2: Jan 30-Feb 5 & 134 & -1 & 10 & 19 & 3 & 0 & -10 & -20 & . & . & 135 \\
\hline Week 3: Feb 6-12 & 159 & -23 & 10 & 24 & 2 & 0 & -10 & -13 & . & . & 149 \\
\hline \multicolumn{12}{|c|}{ 40ft Dry } \\
\hline & \multicolumn{10}{|c|}{ Carrier Availability Category Responses } & Total \\
\hline Current Week: Jan 23-29 & 62 & 30 & 6 & 3 & 0 & -10 & -10 & -15 & . & . & 66 \\
\hline Week 2: Jan 30-Feb 5 & 62 & 30 & 2 & 4 & 0 & 41 & -20 & -48 & . & . & 71 \\
\hline Week 3: Feb 6-12 & 60 & 30 & 1 & 6 & 0 & 27 & -20 & -50 & $\cdot$ & . & 54 \\
\hline \multicolumn{12}{|c|}{ 40ft High Cube } \\
\hline & \multicolumn{10}{|c|}{ Carrier Availability Category Responses } & Total \\
\hline Current Week: Jan 23-29 & 123 & 50 & 25 & 10 & -3 & -8 & -16 & -20 & . & . & 161 \\
\hline Week 2: Jan 30-Feb 5 & 56 & 50 & 7 & 16 & -6 & -8 & -16 & -25 & $\cdot$ & . & 74 \\
\hline Week 3: Feb 6-12 & 72 & 50 & -6 & 16 & -17 & -8 & -20 & -20 & . & . & 67 \\
\hline \multicolumn{12}{|c|}{ 40ft Refrigerated } \\
\hline & \multicolumn{10}{|c|}{ Carrier Availability Category Responses } & Total \\
\hline Current Week: Jan 23-29 & 25 & 0 & . & . & . & . & . & . & . & . & 25 \\
\hline Week 2: Jan 30-Feb 5 & 23 & 0 & . & . & . & . & . & . & . & $\cdot$ & 23 \\
\hline Week 3: Feb 6-12 & 17 & 0 & . & . & . & . & . & . & . & . & 17 \\
\hline \multicolumn{12}{|c|}{ 20ft Refrigerated } \\
\hline & \multicolumn{10}{|c|}{ Carrier Availability Category Responses } & Total \\
\hline Current Week: Jan 23-29 & 1 & . & . & . & . & . & . & . & . & . & 1 \\
\hline Week 2: Jan 30-Feb 5 & 1 & . & . & . & . & . & . & . & $\cdot$ & . & 1 \\
\hline Week 3: Feb 6-12 & 1 & . & . & . & . & . & . & . & . & . & 1 \\
\hline
\end{tabular}

Note: "." denotes no response from carriers.

\begin{tabular}{|l|c|c|}
\hline \multicolumn{3}{|c|}{ Six-Month Snapshot of Average Weekly } \\
Throughput \\
June 2012-November 2012 \\
\hline Container Types & Average Total & Range of Individual \\
& Weekly Throughput* & Carrier Responses \\
\hline 20ft & 171 & $7 . .60$ \\
40ft & 196 & $9 . .75$ \\
40ft High Cubes & 198 & $14 \ldots 59$ \\
40ft Refrigerated & 1 & $0 . . .1$ \\
20ft Refrigerated & 0 & $0 . . .0$ \\
\hline
\end{tabular}

*Data reflect the sum of the individual carriers' average weekly throughput over a 6month timeframe 


\begin{tabular}{|c|c|c|c|c|c|c|c|c|c|c|c|}
\hline \multicolumn{12}{|c|}{ Los Angeles and Long Beach } \\
\hline \multicolumn{12}{|c|}{ 20ft Dry } \\
\hline & \multicolumn{10}{|c|}{ Carrier Availability Category Responses } & Total \\
\hline Current Week: Jan 23-29 & 931 & 769 & 700 & 306 & 287 & 202 & 45 & -43 & . & . & 3,197 \\
\hline Week 2: Jan 30-Feb 5 & 633 & 977 & 700 & 306 & 345 & 316 & 50 & 17 & . & . & 3,344 \\
\hline Week 3: Feb 6-12 & 605 & 1,145 & 700 & 306 & -15 & 474 & 50 & 152 & . & . & 3,417 \\
\hline \multicolumn{12}{|c|}{ 40ft Dry } \\
\hline & \multicolumn{10}{|c|}{ Carrier Availability Category Responses } & Total \\
\hline Current Week: Jan 23-29 & 1,400 & 1,187 & 1,149 & 866 & 487 & 339 & 302 & 45 & . & . & 5,775 \\
\hline Week 2: Jan 30-Feb 5 & 1,400 & 1,549 & 905 & 1,034 & 371 & 339 & 445 & 50 & . & . & 6,093 \\
\hline Week 3: Feb 6-12 & 1,400 & 2,129 & 850 & 1,473 & -17 & 339 & 613 & 50 & . & . & 6,837 \\
\hline \multicolumn{12}{|c|}{ 40ft High Cube } \\
\hline & \multicolumn{10}{|c|}{ Carrier Availability Category Responses } & Total \\
\hline Current Week: Jan 23-29 & 2,500 & 1,733 & 1,198 & 862 & 290 & 60 & -24 & -114 & . & . & 6,505 \\
\hline Week 2: Jan 30-Feb 5 & 2,500 & 1,364 & 1,590 & 862 & 173 & 60 & -5 & -455 & . & . & 6,089 \\
\hline Week 3: Feb 6-12 & 2,500 & 1,320 & 2,105 & 862 & -196 & 60 & 48 & -357 & . & . & 6,342 \\
\hline \multicolumn{12}{|c|}{ 40ft Refrigerated } \\
\hline & \multicolumn{10}{|c|}{ Carrier Availability Category Responses } & Total \\
\hline Current Week: Jan 23-29 & 583 & 311 & 170 & 125 & 93 & 75 & 30 & . & . & . & 1,387 \\
\hline Week 2: Jan 30-Feb 5 & 643 & 321 & 57 & 100 & 106 & 75 & 30 & . & . & . & 1,332 \\
\hline Week 3: Feb 6-12 & 611 & 304 & 30 & 100 & 122 & 75 & 30 & . & . & . & 1,272 \\
\hline \multicolumn{12}{|c|}{ 20ft Refrigerated } \\
\hline & \multicolumn{10}{|c|}{ Carrier Availability Category Responses } & Total \\
\hline Current Week: Jan 23-29 & 44 & 20 & 17 & . & . & . & . & . & . & . & 81 \\
\hline Week 2: Jan 30-Feb 5 & 60 & 20 & 15 & . & . & . & . & . & . & . & 95 \\
\hline Week 3: Feb 6-12 & 75 & 20 & 8 & . & . & . & . & . & . & . & 103 \\
\hline
\end{tabular}

Note: "." denotes no response from carriers.

\begin{tabular}{|l|c|c|}
\hline \multicolumn{3}{|c|}{ Six-Month Snapshot of Average Weekly } \\
Throughput \\
June 2012-November 2012 \\
\hline Container Types & $\begin{array}{c}\text { Average Total } \\
\text { Weekly Throughput* }\end{array}$ & $\begin{array}{c}\text { Range of Individual } \\
\text { Carrier Responses }\end{array}$ \\
\hline $\mathbf{2 0 f t}$ & 3,827 & $46 . .2500$ \\
$\mathbf{4 0 f t}$ & 4,744 & $83 \ldots 3000$ \\
$\mathbf{4 0 f t}$ High Cubes & 7,085 & $203 \ldots 3300$ \\
$\mathbf{4 0 f t}$ Refrigerated & 684 & $21 \ldots 300$ \\
20ft Refrigerated & 64 & $1 \ldots 50$ \\
\hline
\end{tabular}

*Data reflect the sum of the individual carriers' average weekly throughput over a 6 month timeframe 


\section{Memphis, TN}

\begin{tabular}{|c|c|c|c|c|c|c|c|c|c|c|c|}
\hline \multicolumn{12}{|c|}{ Memphis } \\
\hline \multicolumn{12}{|c|}{ 20ft Dry } \\
\hline & \multicolumn{10}{|c|}{ Carrier Availability Category Responses } & Total \\
\hline Current Week: Jan 23-29 & 200 & 68 & 51 & 17 & 16 & 12 & 8 & -73 & . & . & 299 \\
\hline Week 2: Jan 30-Feb 5 & 200 & 46 & 49 & 13 & 16 & 4 & 11 & -88 & . & . & 251 \\
\hline Week 3: Feb 6-12 & 200 & 37 & 81 & 10 & 16 & 0 & 12 & -94 & . & . & 262 \\
\hline \multicolumn{12}{|c|}{ 40ft Dry } \\
\hline & \multicolumn{10}{|c|}{ Carrier Availability Category Responses } & Total \\
\hline Current Week: Jan 23-29 & 200 & 105 & 49 & 29 & 20 & 10 & 10 & -186 & . & . & 237 \\
\hline Week 2: Jan 30-Feb 5 & 200 & 133 & 67 & 16 & 76 & 10 & 8 & -190 & . & . & 320 \\
\hline Week 3: Feb 6-12 & 200 & 186 & 62 & 14 & 143 & 10 & 10 & -196 & . & . & 429 \\
\hline \multicolumn{12}{|c|}{ 40ft High Cube } \\
\hline & \multicolumn{10}{|c|}{ Carrier Availability Category Responses } & Total \\
\hline Current Week: Jan 23-29 & 1,300 & 339 & 121 & 41 & 17 & 16 & 11 & 7 & . & . & 1,852 \\
\hline Week 2: Jan 30-Feb 5 & 1,300 & 453 & 179 & 45 & 4 & 16 & 22 & 8 & . & . & 2,027 \\
\hline Week 3: Feb 6-12 & 1,300 & 570 & 259 & 41 & 2 & 16 & 81 & 6 & . & . & 2,275 \\
\hline \multicolumn{12}{|c|}{ 40ft Refrigerated } \\
\hline & \multicolumn{10}{|c|}{ Carrier Availability Category Responses } & Total \\
\hline Current Week: Jan 23-29 & 17 & 0 & -5 & . & . & . & . & . & . & . & 12 \\
\hline Week 2: Jan 30-Feb 5 & 25 & 0 & -7 & . & . & . & . & . & . & . & 18 \\
\hline Week 3: Feb 6-12 & 31 & 0 & -9 & . & . & . & . & . & . & . & 22 \\
\hline \multicolumn{12}{|c|}{ 20ft Refrigerated } \\
\hline & \multicolumn{10}{|c|}{ Carrier Availability Category Responses } & Total \\
\hline Current Week: Jan 23-29 & -2 & . & . & . & . & . & . & . & . & . & -2 \\
\hline Week 2: Jan 30-Feb 5 & -2 & . & 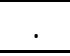 & . & . & . & . & . & . & . & -2 \\
\hline Week 3: Feb 6-12 & -2 & & & . & . & . & . & . & . & . & -2 \\
\hline
\end{tabular}

Note: "." denotes no response from carriers.

\begin{tabular}{|l|c|c|}
\hline \multicolumn{3}{|c|}{ Six-Month Snapshot of Average Weekly } \\
Throughput \\
June 2012-November 2012 \\
\hline Container Types & $\begin{array}{c}\text { Average Total } \\
\text { Weekly Throughput* }\end{array}$ & $\begin{array}{c}\text { Range of Individual } \\
\text { Carrier Responses }\end{array}$ \\
\hline 20ft & 558 & $11 . . .300$ \\
40ft & 430 & $11 . . .200$ \\
40ft High Cubes & 884 & $9 . .600$ \\
40ft Refrigerated & 2 & $0 \ldots 2$ \\
20ft Refrigerated & 0 & $0 . . .0$ \\
\hline
\end{tabular}

*Data reflect the sum of the individual carriers' average weekly throughput over a 6month timeframe 


\section{Minneapolis, MN}

\begin{tabular}{|c|c|c|c|c|c|c|c|c|c|c|c|}
\hline \multicolumn{12}{|c|}{ Minneapolis } \\
\hline \multicolumn{12}{|c|}{ 20ft Dry } \\
\hline & \multicolumn{10}{|c|}{ Carrier Availability Category Responses } & Total \\
\hline Current Week: Jan 23-29 & 169 & 71 & 34 & 20 & 10 & 6 & -2 & -9 & . & . & 299 \\
\hline Week 2: Jan 30-Feb 5 & 134 & 65 & 34 & 19 & 10 & 29 & 4 & -9 & . & . & 286 \\
\hline Week 3: Feb 6-12 & 106 & 82 & 34 & 18 & 10 & 40 & 10 & -8 & . & . & 292 \\
\hline \multicolumn{12}{|c|}{ 40ft Dry } \\
\hline & \multicolumn{10}{|c|}{ Carrier Availability Category Responses } & Total \\
\hline Current Week: Jan 23-29 & 31 & 10 & 8 & 6 & 5 & 2 & -2 & -37 & . & . & 23 \\
\hline Week 2: Jan 30-Feb 5 & 33 & 10 & 7 & 8 & 5 & -47 & -10 & -41 & . & . & -35 \\
\hline Week 3: Feb 6-12 & 14 & 10 & 7 & 6 & 5 & -89 & -10 & -18 & . & . & -75 \\
\hline \multicolumn{12}{|c|}{ 40ft High Cube } \\
\hline & \multicolumn{10}{|c|}{ Carrier Availability Category Responses } & Total \\
\hline Current Week: Jan 23-29 & 65 & 30 & 12 & 11 & 7 & 2 & -10 & -35 & . & . & 82 \\
\hline Week 2: Jan 30-Feb 5 & 85 & 30 & -4 & 11 & -63 & 3 & -10 & -34 & . & . & 18 \\
\hline Week 3: Feb 6-12 & 115 & 30 & -5 & 11 & -136 & 2 & -10 & -38 & . & . & -31 \\
\hline \multicolumn{12}{|c|}{ 40ft Refrigerated } \\
\hline & \multicolumn{10}{|c|}{ Carrier Availability Category Responses } & Total \\
\hline Current Week: Jan 23-29 & 0 & -13 & & & . & . & . & & . & . & -13 \\
\hline Week 2: Jan 30-Feb 5 & 0 & -17 & • & . & . & . & . & . & . & . & -17 \\
\hline Week 3: Feb 6-12 & 0 & -21 & & & . & . & . & & . & . & -21 \\
\hline \multicolumn{12}{|c|}{ 20ft Refrigerated } \\
\hline & \multicolumn{10}{|c|}{ Carrier Availability Category Responses } & Total \\
\hline Current Week: Jan 23-29 & 0 & . & & 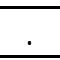 & . & . & . & 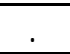 & . & . & 0 \\
\hline Week 2: Jan 30-Feb 5 & 0 & . & & . & . & . & . & 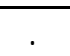 & . & . & 0 \\
\hline Week 3: Feb 6-12 & 0 & . & & & - &. & & & . & . & 0 \\
\hline
\end{tabular}

Note: "." denotes no response from carriers.

\section{Six-Month Snapshot of Average Weekly}

Throughput

June 2012-November 2012

\begin{tabular}{|l|c|c|}
\hline Container Types & $\begin{array}{c}\text { Average Total } \\
\text { Weekly Throughput* }\end{array}$ & $\begin{array}{c}\text { Range of Individual } \\
\text { Carrier Responses }\end{array}$ \\
\hline 20ft & 200 & $3 \ldots 102$ \\
40ft & 181 & $1 . .80$ \\
40ft High Cubes & 223 & $2 \ldots 144$ \\
40ft Refrigerated & 4 & $0 . .4$ \\
20ft Refrigerated & 1 & $0 . .1$ \\
\hline
\end{tabular}

*Data reflect the sum of the individual carriers' average weekly throughput over a 6month timeframe 


\section{New Orleans, LA}

\begin{tabular}{|c|c|c|c|c|c|c|c|c|c|c|c|}
\hline \multicolumn{12}{|c|}{ New Orleans } \\
\hline \multicolumn{12}{|c|}{ 20ft Dry } \\
\hline & \multicolumn{10}{|c|}{ Carrier Availability Category Responses } & Total \\
\hline Current Week: Jan 23-29 & 189 & 121 & 20 & 14 & 10 & 1 & 0 & 0 & . & . & 355 \\
\hline Week 2: Jan 30-Feb 5 & 329 & 93 & 19 & 15 & 5 & 1 & 0 & 0 & . & . & 462 \\
\hline Week 3: Feb 6-12 & 224 & 86 & 38 & 16 & 10 & 2 & 0 & 0 & . & . & 376 \\
\hline \multicolumn{12}{|c|}{ 40ft Dry } \\
\hline & \multicolumn{10}{|c|}{ Carrier Availability Category Responses } & Total \\
\hline Current Week: Jan 23-29 & 107 & 38 & 14 & 4 & 0 & -1 & -3 & -4 & . & . & 155 \\
\hline Week 2: Jan 30-Feb 5 & 120 & 11 & 18 & 0 & 0 & 1 & -6 & -4 & - & . & 140 \\
\hline Week 3: Feb 6-12 & 54 & -6 & 17 & -1 & 0 & 1 & 13 & -4 & . & . & 74 \\
\hline \multicolumn{12}{|c|}{ 40ft High Cube } \\
\hline & \multicolumn{10}{|c|}{ Carrier Availability Category Responses } & Total \\
\hline Current Week: Jan 23-29 & 359 & 48 & 17 & 15 & 4 & 1 & 0 & -2 & . & . & 442 \\
\hline Week 2: Jan 30-Feb 5 & 282 & 21 & 23 & 18 & 17 & 1 & 0 & -2 & . & . & 360 \\
\hline Week 3: Feb 6-12 & 179 & . & 28 & 17 & 11 & 3 & 0 & -2 & . & . & 236 \\
\hline \multicolumn{12}{|c|}{ 40ft Refrigerated } \\
\hline & \multicolumn{10}{|c|}{ Carrier Availability Category Responses } & Total \\
\hline Current Week: Jan 23-29 & 3 & 0 & -8 & . & . & . & . & . & . & . & -5 \\
\hline Week 2: Jan 30-Feb 5 & -2 & 0 & -8 & . & . & . & . & . & . & . & -10 \\
\hline Week 3: Feb 6-12 & -7 & 0 & -8 & . & . & . & . & . & . & . & -15 \\
\hline \multicolumn{12}{|c|}{ 20ft Refrigerated } \\
\hline & \multicolumn{10}{|c|}{ Carrier Availability Category Responses } & Total \\
\hline Current Week: Jan 23-29 & 3 & -1 & . & . & . & . & . & . & . & . & 2 \\
\hline Week 2: Jan 30-Feb 5 & 3 & -11 & . & . & . & . & . & . & . & . & -8 \\
\hline Week 3: Feb 6-12 & 3 & -20 & . & . & . & . & . & . & . & . & -17 \\
\hline
\end{tabular}

Note: "." denotes no response from carriers.

\begin{tabular}{|l|c|c|}
\hline \multicolumn{3}{|c|}{ Six-Month Snapshot of Average Weekly } \\
Throughput \\
June 2012-November 2012 \\
\hline Container Types & $\begin{array}{c}\text { Average Total } \\
\text { Weekly Throughput* }\end{array}$ & $\begin{array}{c}\text { Range of Individual } \\
\text { Carrier Responses }\end{array}$ \\
\hline 20ft & 246 & $5 \ldots 158$ \\
40ft & 157 & $5 \ldots .80$ \\
40ft High Cubes & 221 & $5 \ldots 139$ \\
40ft Refrigerated & 1 & $0 \ldots 1$ \\
20ft Refrigerated & 5 & $0 \ldots 5$ \\
\hline
\end{tabular}

*Data reflect the sum of the individual carriers' average weekly throughput over a 6month timeframe 


\section{New York, NY}

\begin{tabular}{|c|c|c|c|c|c|c|c|c|c|c|}
\hline \multicolumn{11}{|c|}{ New York } \\
\hline \multicolumn{11}{|c|}{ 20ft Dry } \\
\hline & \multicolumn{9}{|c|}{ Carrier Availability Category Responses } & Total \\
\hline Current Week: Jan 23-29 & 456 & 430 & 321 & 300 & 289 & 146 & 129 & 30 & . & 2,101 \\
\hline Week 2: Jan 30-Feb 5 & 778 & 551 & 221 & 300 & 478 & 146 & 183 & 40 & . & 2,697 \\
\hline Week 3: Feb 6-12 & 1,060 & 639 & 201 & 300 & 644 & 146 & 187 & 45 & . & 3,222 \\
\hline \multicolumn{11}{|c|}{ 40ft Dry } \\
\hline & \multicolumn{9}{|c|}{ Carrier Availability Category Responses } & Total \\
\hline Current Week: Jan 23-29 & 864 & 307 & 235 & 200 & 85 & 62 & 25 & -27 & . & 1,751 \\
\hline Week 2: Jan 30-Feb 5 & 892 & 401 & 379 & 200 & 120 & 62 & 35 & 83 & . & 2,172 \\
\hline Week 3: Feb 6-12 & 853 & 472 & 549 & 200 & 110 & 62 & 40 & 83 & . & 2,369 \\
\hline \multicolumn{11}{|c|}{ 40ft High Cube } \\
\hline & \multicolumn{9}{|c|}{ Carrier Availability Category Responses } & Total \\
\hline Current Week: Jan 23-29 & 1,084 & 1,000 & 637 & 304 & 270 & 240 & 109 & 25 & . & 3,669 \\
\hline Week 2: Jan 30-Feb 5 & 1,203 & 1,000 & 838 & 344 & 470 & 240 & 131 & 35 & . & 4,261 \\
\hline Week 3: Feb 6-12 & 1,258 & 1,000 & 1,072 & 397 & 698 & 240 & 120 & 50 & . & 4,835 \\
\hline \multicolumn{11}{|c|}{ 40ft Refrigerated } \\
\hline & \multicolumn{9}{|c|}{ Carrier Availability Category Responses } & Total \\
\hline Current Week: Jan 23-29 & 241 & 148 & 127 & 104 & 50 & 23 & 15 & . & . & 708 \\
\hline Week 2: Jan 30-Feb 5 & 276 & 232 & 174 & 80 & 50 & 23 & 15 &. & . & 850 \\
\hline Week 3: Feb 6-12 & 315 & 339 & 222 & 77 & 50 & 23 & 20 &. & . & 1,046 \\
\hline \multicolumn{11}{|c|}{ 20ft Refrigerated } \\
\hline & \multicolumn{9}{|c|}{ Carrier Availability Category Responses } & Total \\
\hline Current Week: Jan 23-29 & 27 & 10 & -3 & . & . & . & . & . & . & 34 \\
\hline Week 2: Jan 30-Feb 5 & 32 & 10 & -2 & . & . & . & . & . & . & 40 \\
\hline Week 3: Feb 6-12 & 37 & 10 & -2 & 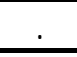 & & . & . & 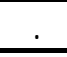 & . & 45 \\
\hline
\end{tabular}

Note: "." denotes no response from carriers.

\begin{tabular}{|l|c|c|}
\hline \multicolumn{3}{|c|}{ Six-Month Snapshot of Average Weekly } \\
Throughput \\
June 2012-November 2012 \\
\hline Container Types & $\begin{array}{c}\text { Average Total } \\
\text { Weekly Throughput* }\end{array}$ & $\begin{array}{c}\text { Range of Individual } \\
\text { Carrier Responses }\end{array}$ \\
\hline 20ft & 714 & $3 \ldots 150$ \\
40ft & 901 & $56 \ldots .250$ \\
40ft High Cubes & 1,539 & $87 . . .400$ \\
40ft Refrigerated & 113 & $0 \ldots 36$ \\
20ft Refrigerated & 17 & $0 \ldots 10$ \\
\hline
\end{tabular}

*Data reflect the sum of the individual carriers' average weekly throughput over a 6month timeframe 


\section{Norfolk, VA}

\section{Norfolk}

\section{$20 \mathrm{ft}$ Dry}

Carrier Availability Category Responses

Total

\begin{tabular}{|l|l|l|l|l|l|l|l|l|l|l|l|}
\hline Current Week: Jan 23-29 & 314 & 200 & 115 & 27 & 20 & 20 & 10 & -6 &. &. & 700 \\
\hline Week 2: Jan 30-Feb 5 & 357 & 208 & 174 & 20 & 20 & 55 & 10 & -6 &. &. & 838 \\
\hline Week 3: Feb 6-12 & 408 & 201 & 207 & 15 & 20 & 47 & 10 & -6 &. &. & 902 \\
\hline \multicolumn{10}{|c|}{ 40ft Dry } \\
\hline
\end{tabular}

Carrier Availability Category Responses

Total

\begin{tabular}{|c|c|c|c|c|c|c|c|c|c|c|c|}
\hline Current Week: Jan 23-29 & 188 & 183 & 130 & 117 & 11 & 11 & -14 & -23 & . & . & 603 \\
\hline Week 2: Jan 30-Feb 5 & 203 & 171 & 130 & 105 & 6 & 11 & -14 & -71 & . & . & 541 \\
\hline Week 3: Feb 6-12 & 215 & 145 & 130 & 148 & 5 & 11 & -14 & -50 & . & . & 590 \\
\hline \multicolumn{12}{|c|}{ 40ft High Cube } \\
\hline & \multicolumn{10}{|c|}{ Carrier Availability Category Responses } & Total \\
\hline Current Week: Jan 23-29 & 529 & 350 & 346 & 31 & 24 & 10 & 10 & -14 & . & . & 1,286 \\
\hline Week 2: Jan 30-Feb 5 & 641 & 350 & 373 & 7 & -2 & -114 & 10 & -14 & . & . & 1,251 \\
\hline Week 3: Feb 6-12 & 765 & 350 & 389 & 5 & 14 & -96 & 10 & -14 & . & . & 1,423 \\
\hline \multicolumn{12}{|c|}{ 40ft Refrigerated } \\
\hline & \multicolumn{10}{|c|}{ Carrier Availability Category Responses } & Total \\
\hline Current Week: Jan 23-29 & 148 & 146 & 74 & 30 & 23 & 1 & -2 & . & . & . & 420 \\
\hline Week 2: Jan 30-Feb 5 & 232 & 57 & 87 & 30 & 6 & 5 & -2 & . & . & . & 415 \\
\hline Week 3: Feb 6-12 & 339 & -29 & 92 & 30 & -25 & 5 & -2 & . & . & . & 410 \\
\hline \multicolumn{12}{|c|}{ 20ft Refrigerated } \\
\hline & \multicolumn{10}{|c|}{ Carrier Availability Category Responses } & Total \\
\hline Current Week: Jan 23-29 & 12 & 1 & -1 & . & . & . & . & . & . & . & 12 \\
\hline Week 2: Jan 30-Feb 5 & 12 & 1 & -1 & . &. & . & . & . & . & . & 12 \\
\hline Week 3: Feb 6-12 & 9 & 1 & -1 & 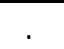 & . & . & 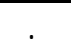 & & . & . & 9 \\
\hline
\end{tabular}

Note: "." denotes no response from carriers.

\section{Six-Month Snapshot of Average Weekly}

Throughput

June 2012-November 2012

\begin{tabular}{|l|c|c|}
\hline Container Types & $\begin{array}{c}\text { Average Total } \\
\text { Weekly Throughput* }\end{array}$ & $\begin{array}{c}\text { Range of Individual } \\
\text { Carrier Responses }\end{array}$ \\
\hline 20ft & 338 & $1 \ldots 175$ \\
40ft & 797 & $8 \ldots 200$ \\
40ft High Cubes & 1,035 & $74 \ldots 300$ \\
40ft Refrigerated & 139 & $0 \ldots 58$ \\
20ft Refrigerated & 6 & $0 . . .5$ \\
\hline
\end{tabular}

*Data reflect the sum of the individual carriers' average weekly throughput over a 6month timeframe 


\section{Oakland, CA}

Oakland

$20 \mathrm{ft}$ Dry

Carrier Availability Category Responses

Total

\begin{tabular}{|l|c|c|c|c|c|c|c|c|c|c|c|}
\hline Current Week: Jan 23-29 & 456 & 414 & 91 & 50 & 32 & 20 & -9 &. &. &. & 1,054 \\
\hline Week 2: Jan 30-Feb 5 & 441 & 450 & 61 & 50 & 32 & 20 & 29 & -26 &. &. & 1,057 \\
\hline Week 3: Feb 6-12 & 392 & 491 & 50 & 50 & 32 & 20 & -17 & -54 &. &. & 964 \\
\hline \multicolumn{10}{|c|}{ 40ft Dry }
\end{tabular}

Carrier Availability Category Responses

Total

\begin{tabular}{|c|c|c|c|c|c|c|c|c|c|c|c|}
\hline Current Week: Jan 23-29 & 350 & 70 & 34 & 29 & 20 & 4 & -76 & -163 & . & . & 268 \\
\hline Week 2: Jan 30-Feb 5 & 256 & 70 & -2 & 35 & 20 & 4 & -2 & -201 &. & . & 180 \\
\hline Week 3: Feb 6-12 & 97 & 70 & -68 & 20 & 20 & 4 & -88 & -243 & . & . & -188 \\
\hline \multicolumn{12}{|c|}{ 40ft High Cube } \\
\hline & \multicolumn{10}{|c|}{ Carrier Availability Category Responses } & Total \\
\hline Current Week: Jan 23-29 & 339 & 289 & 100 & 25 & 25 & -5 & -89 & -139 & . & . & 545 \\
\hline Week 2: Jan 30-Feb 5 & 346 & 133 & 100 & 25 & 20 & -32 & -67 & -158 & . & . & 367 \\
\hline Week 3: Feb 6-12 & 358 & 15 & 100 & 25 & 25 & -63 & -57 & -160 & . & . & 243 \\
\hline \multicolumn{12}{|c|}{ 40ft Refrigerated } \\
\hline & \multicolumn{10}{|c|}{ Carrier Availability Category Responses } & Total \\
\hline Current Week: Jan 23-29 & 350 & 101 & 50 & 20 & -40 & -74 & -84 & -86 & . & . & 237 \\
\hline Week 2: Jan 30-Feb 5 & 332 & 112 & 50 & 20 & -40 & -102 & -99 & -137 & $\cdot$ & . & 136 \\
\hline Week 3: Feb 6-12 & 301 & 73 & 50 & 20 & -40 & -128 & -100 & -175 & . & . & 1 \\
\hline \multicolumn{12}{|c|}{ 20ft Refrigerated } \\
\hline & \multicolumn{10}{|c|}{ Carrier Availability Category Responses } & Total \\
\hline Current Week: Jan 23-29 & 33 & 12 & 10 & 0 & & . & . & - & 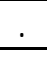 & . & 55 \\
\hline Week 2: Jan 30-Feb 5 & 37 & 8 & 10 & 0 & . & . & . & . & . & . & 55 \\
\hline Week 3: Feb 6-12 & 42 & 5 & 10 & 0 & & . & . & & & . & 57 \\
\hline
\end{tabular}

Note: "." denotes no response from carriers.

\begin{tabular}{|l|c|c|}
\hline \multicolumn{3}{|c|}{ Six-Month Snapshot of Average Weekly } \\
Throughput \\
June 2012-November 2012 \\
\hline Container Types & $\begin{array}{c}\text { Average Total } \\
\text { Weekly Throughput* }\end{array}$ & $\begin{array}{c}\text { Range of Individual } \\
\text { Carrier Responses }\end{array}$ \\
\hline 20ft & 853 & $26 \ldots .250$ \\
40ft & 899 & $14 . .300$ \\
40ft High Cubes & 1,199 & $34 . .400$ \\
40ft Refrigerated & 358 & $26 . .120$ \\
20ft Refrigerated & 24 & $0 . . .8$ \\
\hline
\end{tabular}

*Data reflect the sum of the individual carriers' average weekly throughput over a 6month timeframe 


\section{Savannah, GA}

\begin{tabular}{|c|c|c|c|c|c|c|c|c|c|c|c|}
\hline \multicolumn{12}{|c|}{ Savannah } \\
\hline \multicolumn{12}{|c|}{$20 \mathrm{ft}$ Dry } \\
\hline & \multicolumn{10}{|c|}{ Carrier Availability Category Responses } & Total \\
\hline Current Week: Jan 23-29 & 106 & 100 & 80 & 76 & 75 & 52 & 18 & -10 & . & . & 497 \\
\hline Week 2: Jan 30-Feb 5 & 173 & 100 & 124 & 76 & 117 & 27 & 10 & 31 & . & . & 658 \\
\hline Week 3: Feb 6-12 & 169 & 100 & 171 & 76 & 163 & 25 & 12 & 62 & . & . & 778 \\
\hline \multicolumn{12}{|c|}{ 40ft Dry } \\
\hline & \multicolumn{10}{|c|}{ Carrier Availability Category Responses } & Total \\
\hline Current Week: Jan 23-29 & 301 & 226 & 200 & 20 & 15 & -9 & -105 & -580 & . & . & 68 \\
\hline Week 2: Jan 30-Feb 5 & 454 & 192 & 200 & 20 & 10 & -25 & -38 & -752 & . & . & 61 \\
\hline Week 3: Feb 6-12 & 573 & 179 & 200 & 20 & 10 & -30 & -17 & -923 & . & . & 12 \\
\hline \multicolumn{12}{|c|}{ 40ft High Cube } \\
\hline & \multicolumn{10}{|c|}{ Carrier Availability Category Responses } & Total \\
\hline Current Week: Jan 23-29 & 500 & 288 & 85 & 65 & 61 & 15 & -90 & -524 & . & . & 400 \\
\hline Week 2: Jan 30-Feb 5 & 500 & 405 & 85 & -31 & 16 & 10 & -85 & -701 & . & . & 199 \\
\hline Week 3: Feb 6-12 & 500 & 490 & 85 & -31 & 14 & 11 & -25 & -874 & . & . & 170 \\
\hline \multicolumn{12}{|c|}{ 40ft Refrigerated } \\
\hline & \multicolumn{10}{|c|}{ Carrier Availability Category Responses } & Total \\
\hline Current Week: Jan 23-29 & 91 & 78 & 69 & 61 & 10 & 5 & -3 & . & . & . & 311 \\
\hline Week 2: Jan 30-Feb 5 & 87 & 103 & 74 & -66 & 10 & 5 & -3 & . & . & . & 210 \\
\hline Week 3: Feb 6-12 & 75 & 127 & 104 & -79 & 10 & 8 & -3 & . & . & . & 242 \\
\hline \multicolumn{12}{|c|}{ 20ft Refrigerated } \\
\hline & \multicolumn{10}{|c|}{ Carrier Availability Category Responses } & Total \\
\hline Current Week: Jan 23-29 & 9 & 1 & . & . & . & . & . & . & . & . & 10 \\
\hline Week 2: Jan 30-Feb 5 & 10 & 1 & 2 & . & . &. & . & . & . & . & 13 \\
\hline Week 3: Feb 6-12 & 10 & 1 & 3 & . & . & . & & . & . & . & 14 \\
\hline
\end{tabular}

Note: "." denotes no response from carriers.

\begin{tabular}{|l|c|c|}
\hline \multicolumn{3}{|c|}{ Six-Month Snapshot of Average Weekly } \\
Throughput \\
June 2012-November 2012 \\
\hline Container Types & Average Total & Range of Individual \\
& Weekly Throughput* & Carrier Responses \\
\hline 20ft & 449 & $0 \ldots 150$ \\
40ft & 982 & $0 \ldots 409$ \\
40ft High Cubes & 1,450 & $21 . . .385$ \\
40ft Refrigerated & 221 & $0 . . .80$ \\
20ft Refrigerated & 4 & $0 . .2$ \\
\hline
\end{tabular}

*Data reflect the sum of the individual carriers' average weekly throughput over a 6month timeframe 


\section{Seattle, WA}

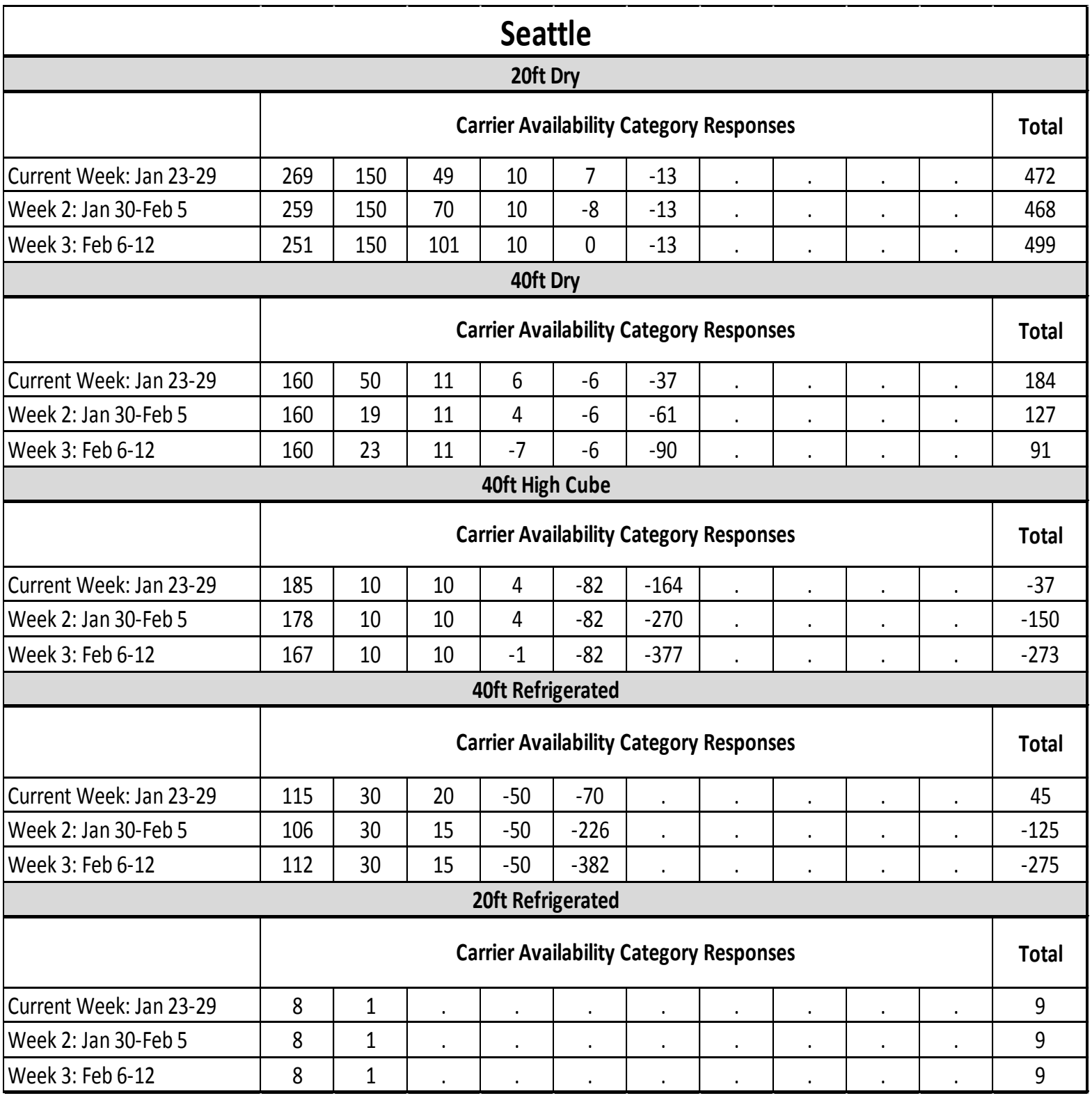

Note: "." denotes no response from carriers.

\begin{tabular}{|l|c|c|}
\hline \multicolumn{3}{|c|}{ Six-Month Snapshot of Average Weekly } \\
Throughput \\
June 2012-November 2012 \\
\hline Container Types & $\begin{array}{c}\text { Average Total } \\
\text { Weekly Throughput* }\end{array}$ & $\begin{array}{c}\text { Range of Individual } \\
\text { Carrier Responses }\end{array}$ \\
\hline 20ft & 172 & $29 . .70$ \\
40ft & 263 & $7 \ldots 91$ \\
40ft High Cubes & 462 & $104 \ldots 148$ \\
40ft Refrigerated & 172 & $0 . .122$ \\
20ft Refrigerated & 7 & $0 . .5$ \\
\hline
\end{tabular}

*Data reflect the sum of the individual carriers' average weekly throughput over a 6month timeframe 


\section{Tacoma, WA}

\begin{tabular}{|c|c|c|c|c|c|c|c|c|c|c|c|}
\hline \multicolumn{12}{|c|}{ Tacoma } \\
\hline \multicolumn{12}{|c|}{$20 \mathrm{ft}$ Dry } \\
\hline & \multicolumn{10}{|c|}{ Carrier Availability Category Responses } & Total \\
\hline Current Week: Jan 23-29 & 85 & 68 & 30 & 10 & 10 & -21 & -23 & . & . & . & 159 \\
\hline Week 2: Jan 30-Feb 5 & 61 & 39 & 30 & 10 & 10 & -34 & -28 & . & . & . & 88 \\
\hline Week 3: Feb 6-12 & 49 & 20 & 30 & 10 & 10 & -58 & -46 & . & . & . & 15 \\
\hline \multicolumn{12}{|c|}{ 40ft Dry } \\
\hline & \multicolumn{10}{|c|}{ Carrier Availability Category Responses } & Total \\
\hline Current Week: Jan 23-29 & 446 & 67 & 10 & 10 & -47 & -87 & -138 & . & . & . & 261 \\
\hline Week 2: Jan 30-Feb 5 & 223 & 69 & 10 & 10 & -47 & -77 & -143 & . & . & . & 45 \\
\hline Week 3: Feb 6-12 & -51 & 45 & 10 & 10 & -47 & -70 & -128 & . & . & . & -231 \\
\hline \multicolumn{12}{|c|}{ 40ft High Cube } \\
\hline & \multicolumn{10}{|c|}{ Carrier Availability Category Responses } & Total \\
\hline Current Week: Jan 23-29 & 60 & 10 & 10 & -55 & -96 & -171 & -349 & . & . & . & -591 \\
\hline Week 2: Jan 30-Feb 5 & -178 & 10 & 10 & -55 & -170 & -229 & -310 & . & . & . & -922 \\
\hline Week 3: Feb 6-12 & -439 & 10 & 10 & -55 & -256 & -249 & -300 & . & . & . & $-1,279$ \\
\hline \multicolumn{12}{|c|}{ 40ft Refrigerated } \\
\hline & \multicolumn{10}{|c|}{ Carrier Availability Category Responses } & Total \\
\hline Current Week: Jan 23-29 & 331 & 290 & 10 & 0 & -34 & -99 & -250 & . & . & . & 248 \\
\hline Week 2: Jan 30-Feb 5 & 299 & 161 & 10 & 0 & -34 & -109 & -232 & . & . & . & 95 \\
\hline Week 3: Feb 6-12 & 281 & 35 & 10 & 0 & -34 & -166 & -200 & . & . & . & -74 \\
\hline \multicolumn{12}{|c|}{ 20ft Refrigerated } \\
\hline & \multicolumn{10}{|c|}{ Carrier Availability Category Responses } & Total \\
\hline Current Week: Jan 23-29 & 6 & 1 & 0 & . & . & . & . & . & . & . & 7 \\
\hline Week 2: Jan 30-Feb 5 & 8 & 1 & 0 & . & . & . & . & . & . & . & 9 \\
\hline Week 3: Feb 6-12 & 4 & 1 & 0 & . & . & . & . & . & . & & 5 \\
\hline
\end{tabular}

Note: "." denotes no response from carriers.

\begin{tabular}{|l|c|c|}
\hline \multicolumn{3}{|c|}{ Six-Month Snapshot of Average Weekly } \\
Throughput \\
June 2012-November 2012 \\
\hline Container Types & Average Total & Range of Individual \\
& Weekly Throughput* & Carrier Responses \\
\hline 20ft & 383 & $1 \ldots 300$ \\
40ft & 671 & $15 . . .500$ \\
40ft High Cubes & 797 & $76 \ldots 600$ \\
40ft Refrigerated & 493 & $0 . .203$ \\
20ft Refrigerated & 4 & $0 . .4$ \\
\hline
\end{tabular}

*Data reflect the sum of the individual carriers' average weekly throughput over a 6month timeframe 


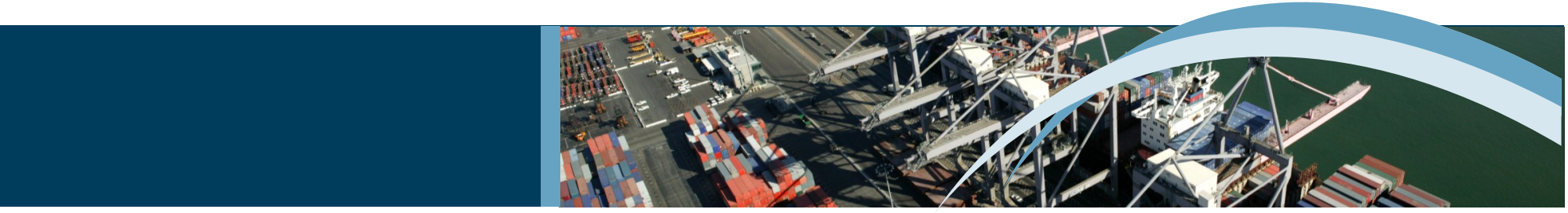

The U.S. Department of Agriculture (USDA) prohibits discrimination in all of its programs and activities on the basis of race, color, national origin, age, disability, and where applicable, sex (including gender identity and expression), marital status, familial status, parental status, religion, sexual orientation, political beliefs, genetic information, reprisal, or because all or part of an individual's income is derived from any public assistance program. (Not all prohibited bases apply to all programs.) Persons with disabilities who require alternative means for communication of program information (Braille, large print, audiotape, etc.) should contact USDA's TARGET Center at (202) 720-2600 (voice and TDD).

To file a complaint of discrimination, write to USDA, Director, Office of Civil Rights, 1400 Independence Avenue, S.W., Washington, D.C. 20250-9410, or call (800) 795-3272 (voice) or (202) 720-6382 (TDD). USDA is an equal opportunity provider and employer.

\section{Preferred citation:}

U.S. Dept. of Agriculture, Agricultural Marketing Service. Ocean Shipping Container Availability Report. January 23, 2013. Web. <http://dx.doi.org/10.9752/TS057.1-23-2013> 